\title{
Circuital analysis of cylindrical structures applied to the electromagnetic resolution of resonant cavities
}

\author{
Felipe L. Penaranda-Foix and Jose M. Catala-Civera \\ ITACA-UPV \\ Camino de Vera, s/n; 46022-Valencia \\ fpenaran@dcom.upv.esEjmcatala@dcom.upv.es \\ Spain
}

\section{Introduction}

The objective of this chapter is first to describe the generalized circuital analysis as a method to solve complex electromagnetic problems and second to apply this specific technique to the determination of the resonant frequency and Q-factor of a cylindrical cavity loaded with a dielectric material when the material is introduced inside the cavity through a hole in the upper wall.

The generalized circuital analysis as a method for solving electromagnetic problems consisting of the segmentation of the whole geometry of the microwave circuit into simpler structures which resolution can be solved in a easier way. Once the simpler structures have been solved separately, they can be joined or combined in order to give the complete solution of the complex structure.

The resolution of the resonant frequency and Quality factor of a coaxially loaded circular cavity with a dielectric material is very interesting, for instance, for the determination of the dielectric properties (complex permittivity) of materials on this type of cavities. This type of analysis on these cavities can be found in the technical literature but in all cases, the effect of the hole to introduce the dielectric material inside the cavity is neglected and in some cases, such as it will be shown in the second part of the chapter, the effect of the hole can introduce considerable errors in the determination of the resonant frequency and quality factor whose can interfere the precision of the permittivity calculations.

In next sections, the effect of the hole for the introduction of dielectric materials inside circular cavities is evaluated by solving the structure by the generalized circuital analysis. Several measurements of circular cavities with dielectric materials will confirm the effect of the hole in the precision of permittivity calculations. 


\section{Circuital Analysis}

In this section the ability of solving complex electromagnetic problems by segmenting the whole structure in several much simpler sub-structures that can be analyzed separately is described. The Generalized Scattering Matrix is also introduced as the method to interconnect (combine) networks with different ports and therefore re-combine the segmented networks to characterize the original structure.

\subsection{The Generalized Admittance Matrix}

The circuital analysis is based on the wave impedance concept. This parameter establishes the relationship between electric and magnetic fields in each point of the space and reflects the interaction between fields and the medium. The concept of impedance (or admittance), associated to each point in a coordinates system, can be extended to the characterization of a given volume by the introduction of the Generalized Admittance Matrix (GAM) ${ }^{1}$ or the Generalized Impedance Matrix (GIM).

As introduced by Schelkunoff in the 30ths and as indicated in (Harrington, 1961), in electromagnetic theories, the relation between the electric field components $\overrightarrow{\mathbf{E}}$ and magnetic field components $\overrightarrow{\mathbf{H}}$ is called as wave impedances. These values are punctual because they are associated to each space point. Then, if the wave impedance characterizes a unique relation between $\overrightarrow{\mathbf{E}}$ and $\overrightarrow{\mathbf{H}}$, the GAM characterizes all the volume free of sources by means of the relationship established by $\overrightarrow{\mathbf{E}}$ and $\overrightarrow{\mathbf{H}}$ on the surrounding surface.

The generalized admittance is unique as probed by the Uniqueness theorem (Harrington, 1961). This theorem establishes that the field inside a closed and lossy region can be completely determined by knowing the sources into the region and the electric and magnetic tangential field components in a fictitious surface that surrounds the region of interest.

Figure 1 shows the schematic of this situation. Region 2 is the region of interest free of sources and region 1 is the region that contains the sources. The Uniqueness theorem guaranties that it is not necessary to know the real sources of region 1 to determine in a unique way the fields $\overrightarrow{\mathbf{E}}$ and $\overrightarrow{\mathbf{H}}$ in any point within region 2 but it is enough to know the tangential electric field $\overrightarrow{\mathbf{E}}_{t}$ in the surface $S$, or the tangential magnetic field $\overrightarrow{\mathbf{H}}_{t}$ in the surface $S$ or the tangential electric field $\overrightarrow{\mathbf{E}}_{t}$ in part of the surface $S$ and the tangential magnetic field $\overrightarrow{\mathbf{H}}_{t}$ in the rest of the surface $S$.

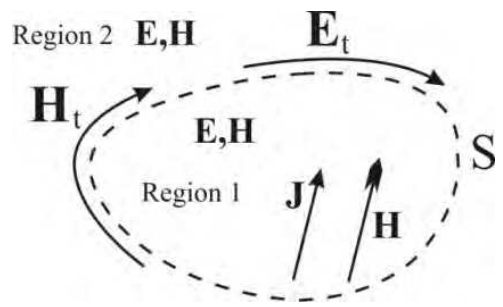

Fig. 1. Generic Surface with two types of regions: with and without sources

\footnotetext{
${ }^{1}$ In an equivalent manner we can speak of Generalized Impedance Matrix (GIM)
} 
Therefore, it is clear that there exists a relationship between both tangential electric and magnetic field components, since the knowledge of one of them ensures the knowledge of all the rest electromagnetic field components. Moreover this relationship is unique due to the Uniqueness Theorem.

The relation between electric and magnetic fields can be expressed as a linear combination of base function according to the following expressions:

$$
\overrightarrow{\mathbf{E}}_{t}=\sum_{n} e_{n} \cdot \overrightarrow{\mathbf{E}}_{n} \quad ; \quad \overrightarrow{\mathbf{H}}_{t}=\sum_{n} h_{n} \cdot \overrightarrow{\mathbf{H}}_{n}
$$

where $\overrightarrow{\mathbf{E}}_{n}$ and $\overrightarrow{\mathbf{H}}_{n}$ are the base functions for the electric and magnetic fields, respectively, and the terms $e_{n}$ and $h_{n}$ are, respectively, the amplitudes of weight of each base function. According to these expressions, the relation between both field components is established by a matrix. This matrix relates the weights of the magnetic field series $h_{n}$ with the weights of the electric field series $e_{n}$. This matrix is known as the Generalized Admittance Matrix $\overline{\overline{\mathbf{Y}}}$. The inverse is called the Generalized impedance Matrix $\overline{\overline{\mathbf{Z}}}$ :

$$
\overrightarrow{\mathbf{h}}=\left(\begin{array}{c}
h_{1} \\
h_{2} \\
\ldots \\
h_{N}
\end{array}\right)=\left(\begin{array}{cccc}
Y_{11} & Y_{12} & \ldots & Y_{1 N} \\
Y_{21} & Y_{22} & \ldots & Y_{2 N} \\
\ldots & \ldots & \ldots & \ldots \\
Y_{N 1} & Y_{N 2} & \ldots & Y_{N N}
\end{array}\right) \cdot\left(\begin{array}{c}
e_{1} \\
e_{2} \\
\ldots \\
e_{N}
\end{array}\right)=\overline{\mathbf{Y}} \cdot \overrightarrow{\mathbf{e}} \quad ; \quad \overrightarrow{\mathbf{e}}=\overline{\mathbf{Z}} \cdot \overrightarrow{\mathbf{h}}
$$

The relation between both matrices $\overline{\overline{\mathbf{Y}}}$ y $\overline{\overline{\mathbf{Z}}}$ is clearly:

$$
\overline{\bar{Z}}=(\overline{\bar{Y}})^{-1}
$$

$N$ refers to the number of terms considered in the series development of the electric and magnetic fields of equation (1).

The definition of this matrix is very important for solving complex electromagnetic problems because it permits to segment the whole structure into simpler structures by making use of circuital theories. The segmentation concept can be first attributed to Harrington en (Harrington, 1961) where diverse waveguide apertures were analyzed by dividing the structure in two parts by placing at the aperture some equivalent currents. This technique was known as Generalized Circuital Formulation. From this first attempts, there has been many references that made use of it. For instance, (Collin, 1966), (Collin, 1991) and (Pozar, 1990) applied this technique for solving cavities excited by slots. Another interesting example is the work of (Gentili \& Melloni, 1996). Some other examples can be found in (Alessandri et al, 1994), (Gimeno \& Guglielmi, 1997) y (Rebollar et al., 1994) for closed structures and in (Valero-Nogueira 1997), (Penaranda-Foix, 2001), (Penaranda-Foix \& FerrandoBataller, 2003), (Penaranda-Foix et al., 2007a) y (Penaranda-Foix et al., 2009) for open problems. 
Figure 2a shows a generic example for illustrating how the circuital theory can be applied to solve complex structures. Figure 2a distinguishes two zones: region $a$ free of sources and region $b$ with sources.

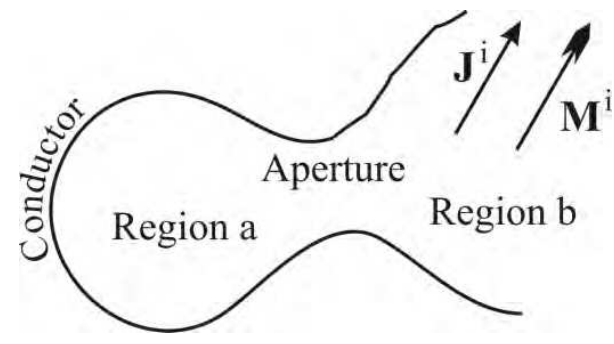

Fig. 2a. Segmentation problem: Two regions connected by the aperture

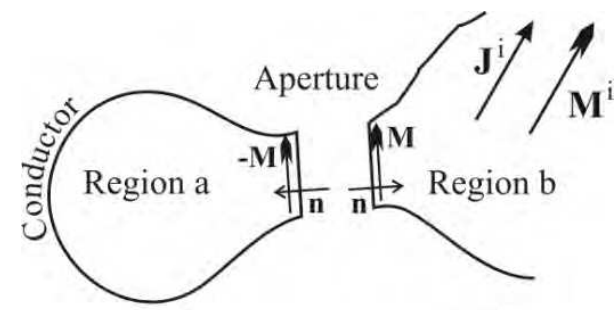

(a)

Fig. 2b. Segmentation problem: Two regions separated by the aperture

Figure $2 \mathrm{~b}$ shows the same structure but segmented in two equivalent problems by means of the equivalent principle. This separation of regions is carried out by placing an electric wall in the aperture between regions $a$ and $b$.

The fields generated in region $b$ are the result of the sources $\mathbf{J}^{i}$ y $\mathbf{M}^{i}$ and the fields created by the equivalent magnetic current $\overrightarrow{\mathbf{M}}=\overrightarrow{\mathbf{E}} \times \hat{\mathbf{n}}$ on the aperture surface. Since the aperture is covered by an electric wall, it is not necessary the use of equivalent electric currents. On the other hand, in region $a$, the field is uniquely created by the magnetic current $-\overrightarrow{\mathbf{M}}$ at the aperture since there are no sources in this region (a).

To raise this problem, continuity of tangential magnetic fields in the aperture is imposed. The total magnetic field on the aperture zone in region $b$ is the summation of the field due to the impressed currents $\overrightarrow{\mathbf{H}}_{t}^{i}$ and the field due to the equivalent sources $\overrightarrow{\mathbf{M}}$ called as $\mathbf{H}_{t}^{b}(\overrightarrow{\mathbf{M}})$ from now on, then:

$$
\overrightarrow{\mathbf{H}}_{t}^{b}=\overrightarrow{\mathbf{H}}_{t}^{i}+\mathbf{H}_{t}^{b}(\overrightarrow{\mathbf{M}})
$$

It is important to remark that both $\overrightarrow{\mathbf{H}}_{t}^{i}$ and $\mathbf{H}_{t}^{b}(\overrightarrow{\mathbf{M}})$ are fields calculated by assuming the aperture as an electric wall.

In a similar manner, the tangential magnetic field in the aperture of region $b$ is due to the equivalent sources $-\overrightarrow{\mathbf{M}}$ called $\mathbf{H}_{t}^{a}(-\overrightarrow{\mathbf{M}})$ from now on, leading to:

$$
\overrightarrow{\mathbf{H}}_{t}^{a}=\mathbf{H}_{t}^{a}(-\overrightarrow{\mathbf{M}})
$$

Where once again $\mathbf{H}_{t}^{a}(-\overrightarrow{\mathbf{M}})$ is calculated assuming the source $-\overrightarrow{\mathbf{M}}$ in the aperture where the electric wall is located.

Making both tangential components equal, we have: 


$$
\begin{gathered}
\overrightarrow{\mathbf{H}}_{t}^{b}=\overrightarrow{\mathbf{H}}_{t}^{i}+\mathbf{H}_{t}^{b}(\overrightarrow{\mathbf{M}})=\overrightarrow{\mathbf{H}}_{t}^{a}=\mathbf{H}_{t}^{a}(-\overrightarrow{\mathbf{M}}) \\
\Downarrow \\
\mathbf{H}_{t}^{b}(\overrightarrow{\mathbf{M}})-\mathbf{H}_{t}^{a}(-\overrightarrow{\mathbf{M}})=-\overrightarrow{\mathbf{H}}_{t}^{i}
\end{gathered}
$$

Equation (6) is the basic equation for the calculation of $\overrightarrow{\mathbf{M}}$ by assuming it is known the operator that relates magnetic fields with the sources in each region.

To solve this equation two numerical methods, very similar between them, are available: The Method of Moments (MoM), see (Harrington, 1967) y (Harrington, 1993), and Modal Analysis or Mode Matching, see (Wexler, 1967).

Assuming that the unknown source $\overrightarrow{\mathbf{M}}$ can be written as a series of base functions, in a similar manner to equation (1),

$$
\overrightarrow{\mathbf{M}}=\sum_{n} v_{n} \cdot \overrightarrow{\mathbf{m}}_{n}
$$

where coefficients $v_{n}$ need to be determined. Substituting this series development in equation (6) and making use of the operator linearity, we can write:

$$
\sum_{n} v_{n} \cdot \overrightarrow{\mathbf{H}}_{t}^{b}\left(\overrightarrow{\mathbf{m}}_{n}\right)-\sum_{n} v_{n} \cdot \overrightarrow{\mathbf{H}}_{t}^{a}\left(-\overrightarrow{\mathbf{m}}_{n}\right)=-\overrightarrow{\mathbf{H}}_{t}^{i}
$$

With the definition of the internal product:

$$
\langle\overrightarrow{\mathbf{A}}, \overrightarrow{\mathbf{B}}\rangle=\iint_{\text {Aperture }}^{\overrightarrow{\mathbf{A}} \cdot \overrightarrow{\mathbf{B}}} \cdot d S
$$

and a set of weight functions $\left\{\overrightarrow{\mathbf{w}}_{n}\right\}$, that in general may differ from the base functions $\overrightarrow{\mathbf{m}}_{n}$, can be applied in (8), leading, thanks to the linearity of the product function, to:

$$
\sum_{n} v_{n} \cdot\left\langle\overrightarrow{\mathbf{w}}_{m}, \overrightarrow{\mathbf{H}}_{t}^{b}\left(\overrightarrow{\mathbf{m}}_{n}\right)\right\rangle-\sum_{n} v_{n} \cdot\left\langle\overrightarrow{\mathbf{w}}_{m},-\overrightarrow{\mathbf{H}}_{t}^{a}\left(\overrightarrow{\mathbf{m}}_{n}\right)\right\rangle=-\left\langle\overrightarrow{\mathbf{w}}_{m}, \overrightarrow{\mathbf{H}}_{t}^{i}\right\rangle
$$

If previous equations are re-written in a matricial form, a new set of matrices $\overline{\overline{\mathbf{Y}}}^{a}$ and $\overline{\overline{\mathbf{Y}}}^{b}$ are defined as the matrices that characterizes the regions $a$ and $b$, respectively.

$$
\left.Y^{a}\right|_{m n}=-\left\langle\overrightarrow{\mathbf{w}}_{m},-\overrightarrow{\mathbf{H}}_{t}^{a}\left(\overrightarrow{\mathbf{m}}_{n}\right)\right\rangle=\left\langle\overrightarrow{\mathbf{w}}_{m}, \overrightarrow{\mathbf{H}}_{t}^{a}\left(\overrightarrow{\mathbf{m}}_{n}\right)\right\rangle ;\left.Y^{b}\right|_{m n}=\left\langle\overrightarrow{\mathbf{w}}_{m}, \overrightarrow{\mathbf{H}}_{t}^{b}\left(\overrightarrow{\mathbf{m}}_{n}\right)\right\rangle
$$

Matrix $\overrightarrow{\mathbf{I}}^{i}$ of dimensions (Mx1) is also defined as,

$$
\left.I^{i}\right|_{m}=\left\langle\overrightarrow{\mathbf{w}}_{m}, \overrightarrow{\mathbf{H}}_{t}^{i}\right\rangle
$$

and the column vector $\overrightarrow{\mathbf{V}}$, of dimensions $(\mathrm{Nx} 1)$, as: 


$$
\left.V\right|_{n}=v_{n}
$$

On the other hand, equation (10) is now:

$$
\left(\overline{\overline{\mathbf{Y}}}^{b}+\overline{\overline{\mathbf{Y}}}^{a}\right) \cdot \overrightarrow{\mathbf{V}}=\overrightarrow{\mathbf{I}}^{i}
$$

$\mathrm{T}$

hen, magnetic current $\overrightarrow{\mathbf{M}}$ is -see equations (6) and (8)-:

$$
\overrightarrow{\mathbf{V}}=\left(\overline{\overline{\mathbf{Y}}}^{b}+\overline{\overline{\mathbf{Y}}}^{a}\right)^{-1} \cdot \overrightarrow{\mathbf{I}}^{i}
$$

It is important to emphasize that equation (15) presents a clear circuital interpretation which is showed in next figure 3 .

Fig. 3. Circuital interpretation of equation (15)

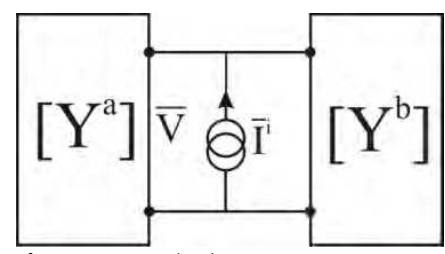

Figure 3 represents two networks, each one characterized by an admittance matrix $\left(\overline{\overline{\mathbf{Y}}}^{a}\right.$ and $\overline{\bar{Y}}^{b}$, respectively) and a current source $\overrightarrow{\mathbf{I}}^{i}$, which solution is given by equation (15). The importance of this result falls in the possibility to analyze and solve each region separately and irrespective of the other regions. Moreover, this result can be extended to networks with more port numbers, as described in (Penaranda-Foix, 2001)

\subsection{The Generalized Scattering Matrix}

In previous section, GAM and GIM matrices were introduced. Next section adds to these matrices the Generalized Scattering Matrix (GSM).

It is well known that by considering a canonic plane as the access port to the network under analysis, the electric and magnetic fields in such surface can be decomposed in forward and reflected waves in the network, respectively. This is especially evident when the wave equation is solved (Balanis, 1989), (Harrington, 1961)-.

Therefore, electric and magnetic fields in a port of the network, can be written as:

$$
\left\{\begin{array}{c}
\overrightarrow{\mathbf{E}}=\overrightarrow{\mathbf{E}}^{(i)}+\overrightarrow{\mathbf{E}}^{(r)} \\
\overrightarrow{\mathbf{H}}=\overrightarrow{\mathbf{H}}^{(i)}-\overrightarrow{\mathbf{H}}^{(r)}=\overline{\overline{\mathbf{Y}}}_{0}^{(i)} \cdot \overrightarrow{\mathbf{E}}^{(i)}-\overline{\overline{\mathbf{Y}}}_{0}^{(r)} \cdot \overrightarrow{\mathbf{E}}^{(r)}
\end{array}\right.
$$

By making use the known concept of characteristic admittances $\overline{\overline{\mathbf{Y}}}_{0}^{(i)}$ y $\overline{\overline{\mathbf{Y}}}_{0}^{(r)}$. 
At the same time, according to equation (1), electric and magnetic fields can be written as a series development. Therefore, the Generalized Scattering Matrix (GSM) can be defined as the relation of the forward electric wave fields $\overrightarrow{\mathbf{E}}^{(i)}$ and the reflected wave fields $\overrightarrow{\mathbf{E}}^{(r)}$ :

$$
\overrightarrow{\mathbf{E}}^{(r)}=\overline{\overline{\mathbf{S}}} \cdot \overrightarrow{\mathbf{E}}^{(i)}
$$

The relationship between the $\overline{\overline{\mathbf{S}}}$ matrix and the admittance $\overline{\overline{\mathbf{Y}}}$ and impedance $\overline{\overline{\mathbf{Z}}}$ matrices is calculated by the following equations

$$
\begin{aligned}
& \overrightarrow{\mathbf{H}}=\overrightarrow{\mathbf{H}}^{(i)}-\overrightarrow{\mathbf{H}}^{(r)}=\overline{\overline{\mathbf{Y}}}_{0}^{(i)} \cdot \overrightarrow{\mathbf{E}}^{(i)}-\overline{\overline{\mathbf{Y}}}_{0}^{(r)} \cdot \overrightarrow{\mathbf{E}}^{(r)}=\overline{\overline{\mathbf{Y}}} \cdot \overrightarrow{\mathbf{E}}=\overline{\overline{\mathbf{Y}}} \cdot\left(\overrightarrow{\mathbf{E}}^{(i)}+\overrightarrow{\mathbf{E}}^{(r)}\right) \\
& \left(\overline{\overline{\mathbf{Y}}}+\overline{\overline{\mathbf{Y}}}_{0}^{(r)}\right) \cdot \overrightarrow{\mathbf{E}}^{(r)}=\left(\overline{\overline{\mathbf{Y}}}_{0}^{(i)}-\overline{\overline{\mathbf{Y}}}\right) \cdot \overrightarrow{\mathbf{E}}^{(i)} \Rightarrow \overrightarrow{\mathbf{E}}^{(r)}=\left(\overline{\overline{\mathbf{Y}}}+\overline{\overline{\mathbf{Y}}}_{0}^{(r)}\right)^{-1} \cdot\left(\overline{\overline{\mathbf{Y}}}_{0}^{(i)}-\overline{\overline{\mathbf{Y}}}\right) \cdot \overrightarrow{\mathbf{E}}^{(i)} \\
& \Downarrow \\
& \overline{\overline{\mathbf{S}}}=\left(\overline{\overline{\mathbf{Y}}}+\overline{\overline{\mathbf{Y}}}_{0}^{(r)}\right)^{-1} \cdot\left(\overline{\overline{\mathbf{Y}}}_{0}^{(i)}-\overline{\overline{\mathbf{Y}}}\right)
\end{aligned}
$$

In a similar manner, the admittance matrix is given by:

$$
\overline{\overline{\mathbf{Y}}}=\left(\overline{\overline{\mathbf{Y}}}_{0}^{(i)}-\overline{\overline{\mathbf{Y}}}_{0}^{(r)} \cdot \overline{\overline{\mathbf{S}}}\right)^{-1} \cdot(\overline{\overline{\mathbf{I}}}+\overline{\overline{\mathbf{S}}})=\overline{\overline{\mathbf{Z}}}^{-1}
$$

\subsection{Connecting networks}

To conclude with this section, the procedure to interconnect networks of several ports and set the new $\overline{\overline{\mathbf{S}}}, \overline{\overline{\mathbf{Y}}}$ o $\overline{\overline{\mathbf{Z}}}$ matrices is described.

Assuming two generic networks characterized by their respective admittance matrices $\overline{\overline{\mathbf{Y}}}^{(1)}$ and $\overline{\overline{\mathbf{Y}}}^{(2)}$ where, for example, the first network presents 5 ports and the second 6 ports, respectively. (See figure 4a for more details of the networks and port numbers).

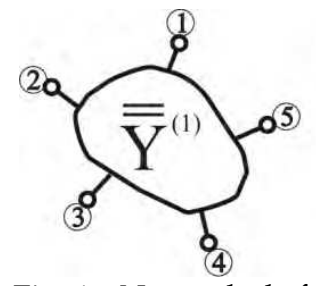

Fig. 4a. Networks before connecting

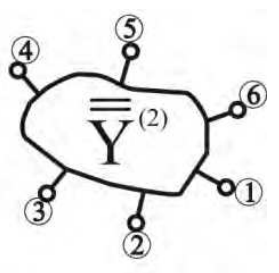

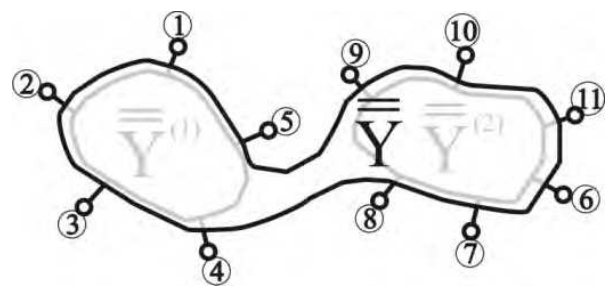

Fig. 4b. Networks joined but not connected

The first step consists of setting a global network with a number of ports equal to the sum of the number of ports of both networks and with a new numeration of ports. (It is a good practice to keep the order of the ports with the new numeration) Thus, the new GAM is witten as: 


$$
\overline{\overline{\mathbf{Y}}}=\left(\begin{array}{cc}
\overline{\overline{\mathbf{Y}}}^{(1)} & \overline{\overline{\mathbf{0}}} \\
\overline{\overline{\mathbf{0}}} & \overline{\overline{\mathbf{Y}}}^{(2)}
\end{array}\right)
$$

Figure $4 \mathrm{~b}$ shows this new network with a commoun numeration of ports. This particular example of figure 4 can be extended to a any network with $N$ ports, as the network showed in figure 5 a.

The second step consist of connecting two (or more) ports from the new network of figure $5 \mathrm{~b}$. By connecting these two ports, for instance ports $l$ and $k$, the resulting network reduces the number of ports to $\mathrm{N}-2$.

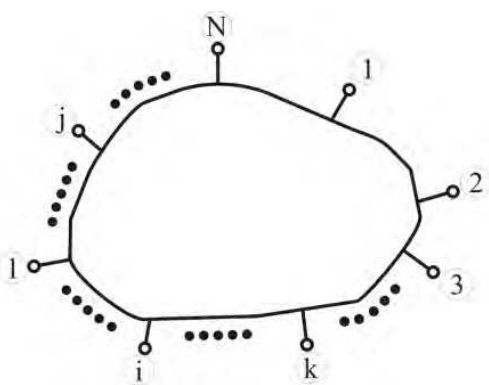

Fig. 5a. Generic network before connecting ports $l$ and $k$.

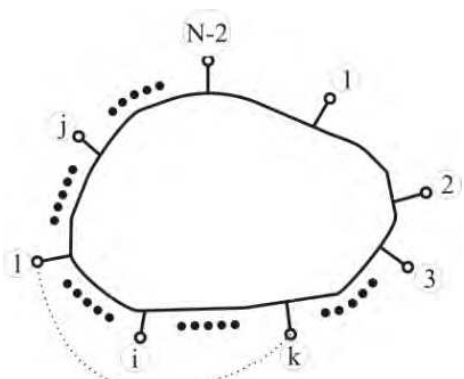

Fig. 5b. Generic network after connecting ports $l$ and $k$.

The new GAM matrix with $\mathrm{N}-2$ ports is calculated by imposing continuity of electric and magnetic tangential component fields between ports $l$ and $k$.

$$
\begin{gathered}
\overrightarrow{\mathbf{h}}_{k}=\sum_{j=1}^{N} \overline{\overline{\mathbf{Y}}}_{k j} \cdot \overrightarrow{\mathbf{e}}_{j}=\overrightarrow{\mathbf{h}}_{l}=\sum_{j=1}^{N} \overline{\overline{\mathbf{Y}}}_{l j} \cdot \overrightarrow{\mathbf{e}}_{j} \\
\Downarrow\left\langle\overrightarrow{\mathbf{e}}_{l}=\overrightarrow{\mathbf{e}}_{k}\right\rangle \\
\sum_{j=1}^{N}\left(\overline{\overline{\mathbf{Y}}}_{k j}-\overline{\overline{\mathbf{Y}}}_{l j}\right) \cdot \overrightarrow{\mathbf{e}}_{j}=\left(\overline{\overline{\mathbf{Y}}}_{l k}+\overline{\overline{\mathbf{Y}}}_{l l}-\overline{\overline{\mathbf{Y}}}_{k l}-\overline{\overline{\mathbf{Y}}}_{k k}\right) \cdot \overrightarrow{\mathbf{e}}_{k}
\end{gathered}
$$

And operating on previous equation, the new GAM matrix with $N-2$ ports, called $\overline{\overline{\mathbf{Y}}}^{(T)}$, is reached:

$$
\left.\overline{\overline{\mathbf{Y}}}_{p q}^{(T)}\right|_{\substack{1 \leq p \leq(N-2) \\ 1 \leq q \leq(N-2)}}=\left.\overline{\overline{\mathbf{Y}}}_{p q}\right|_{\substack{1 \leq p \leq(N-2) \\ 1 \leq q \leq(N-2)}}+\left(\overline{\overline{\mathbf{Y}}}_{p k}+\overline{\overline{\mathbf{Y}}}_{p l}\right) \cdot\left(\overline{\overline{\mathbf{Y}}}_{l k}+\overline{\overline{\mathbf{Y}}}_{l l}-\overline{\overline{\mathbf{Y}}}_{k l}-\overline{\overline{\mathbf{Y}}}_{k k}\right)^{-1} \cdot\left(\overline{\overline{\mathbf{Y}}}_{k q}-\overline{\overline{\mathbf{Y}}}_{l q}\right)
$$

Operating in a similar manner, the impedance matrix GIM, is also found as:

$$
\left.\overline{\overline{\mathbf{Z}}}_{p q}^{(T)}\right|_{\substack{1 \leq p \leq(N-2) \\ 1 \leq q \leq(N-2)}}=\left.\overline{\overline{\mathbf{Z}}}_{p q}\right|_{\substack{1 \leq p \leq(N-2) \\ 1 \leq q \leq(N-2)}}+\left(\overline{\overline{\mathbf{Z}}}_{p k}+\overline{\overline{\mathbf{Z}}}_{p l}\right) \cdot\left(\overline{\overline{\mathbf{Z}}}_{l k}+\overline{\overline{\mathbf{Z}}}_{l l}-\overline{\overline{\mathbf{Z}}}_{k l}-\overline{\overline{\mathbf{Z}}}_{k k}\right)^{-1} \cdot\left(\overline{\overline{\mathbf{Z}}}_{k q}-\overline{\overline{\mathbf{Z}}}_{l q}\right)
$$


Also by imposing the same continuity of tangential fields, the new GSM matrix is obtained:

where:

$$
\begin{aligned}
\left.\overline{\overline{\mathbf{S}}}_{p q}^{(T)}\right|_{\substack{1 \leq p \leq(N-2) \\
1 \leq q \leq(N-2)}}=\left.\overline{\overline{\mathbf{S}}}_{p q}\right|_{\substack{1 \leq p \leq(N-2) \\
1 \leq q \leq(N-2)}}+\overline{\overline{\mathbf{S}}}_{p l} \cdot\left(\overline{\overline{\mathbf{I}}}-\overline{\overline{\mathbf{\Delta}}}_{k l} \cdot \overline{\overline{\mathbf{S}}}_{k k} \cdot \overline{\overline{\mathbf{\Delta}}}_{l k} \cdot \overline{\overline{\mathbf{S}}}_{l l}\right)^{-1} \cdot \overline{\overline{\mathbf{\Delta}}}_{k l} \cdot\left(\overline{\overline{\mathbf{S}}}_{k q}+\overline{\overline{\mathbf{S}}}_{k k} \cdot \overline{\overline{\mathbf{\Delta}}}_{l k} \cdot \overline{\overline{\mathbf{S}}}_{l q}\right)+ \\
+\overline{\overline{\mathbf{S}}}_{p k} \cdot\left(\overline{\overline{\mathbf{I}}}-\overline{\overline{\boldsymbol{\Delta}}}_{l k} \cdot \overline{\overline{\mathbf{S}}}_{l l} \cdot \overline{\overline{\boldsymbol{\Delta}}}_{k l} \cdot \overline{\overline{\mathbf{S}}}_{k k}\right)^{-1} \cdot \overline{\overline{\boldsymbol{\Delta}}}_{l k} \cdot\left(\overline{\overline{\mathbf{S}}}_{l q}+\overline{\overline{\mathbf{S}}}_{l l} \cdot \overline{\overline{\boldsymbol{\Delta}}}_{k l} \cdot \overline{\overline{\mathbf{S}}}_{k q}\right)+
\end{aligned}
$$

$$
\overline{\overline{\mathbf{\Delta}}}_{k l}=\left(\overline{\overline{\mathbf{I}}}-\overline{\overline{\mathbf{S}}}_{k l}\right)^{-1} ; \quad \overline{\overline{\boldsymbol{\Delta}}}_{l k}=\left(\overline{\overline{\mathbf{I}}}-\overline{\overline{\mathbf{S}}}_{l k}\right)^{-1}
$$

Equations (22), (23) and (24) are a powerful set of equations that allow to apply the circuital method to electromagnetic problems by the segmentation procedure.

Once each simple problem or circuit has been analysed and its GAM, GIM or GSM is known, just using the previous equations the whole GAM, GIM or GSM or the orginal problem is calculated.

In the next point this general procedure will be applied to a specific problem: a cylindrical cavity with insertion hole.

\section{Cylindrical cavity analysis}

As an example of the circuital theory described in previous section we will analyze the circular resonant cavities used for the electromagnetic characterization of materials.

To determine accurately the dielectric and magnetic properties of materials is essential nowadays in applications such antennas, radomes, planar circuits, etc. Special attention must be dedicated to the new materials developed for special applications. But not only in those cases: in applications such as microwave heating, where losses associated to the materials determine its capability to be heated, where the measured electric variations will allow to determine how the monitorized material is changing its properties in real time. Finally, a large number of applications can be thought in the world of medicine, to get images, or even from the security view point, in order to know the electromagnetic properties of potentially dangerous materials.

One of the most widely method to determine the dielectric properties is the coaxially filled circular resonant cavity with an insertion hole in the top or in top and in the bottom, to introduce the material. These cavities are based on the use of the resonant mode $\mathrm{TM}_{010}$, with no angular changes -see (Balanis, 1989), (Chen et al., 2004) and (Metaxas \& Meredith, 1988)and in the property that the insertion hole is considered as a cylindrical waveguide under cut-off so there is no propagation along it. Then the cavity, even open, can be considered as a closed cavity.

The problem geometry is shown in figure $6 a$, being figure $6 \mathrm{~b}$ a section, where we can see a cavity of radius $b$ and height $h$ with a generic material of permittivity $\varepsilon_{r 2}$ and permeability $\mu_{r 2}$ (both will be in general air: $\varepsilon_{r 2}=\mu_{r 2}=1$ ). In the center there is an insertion hole, or tube, whose radius is $a$ and its height is larger than the cavity height. It contains the material to be measured with permittivity $\varepsilon_{r 1}$ and permeability $\mu_{r 1}$. Finally, above the measured material there is another one with permittivity $\varepsilon_{r}$ and permeability $\mu_{r}$ (in general air again: $\varepsilon_{r}=\mu_{r}=1$ ). But it is important to remember that the tube is under cut-off and then there is no leakage through it and it is negligible. 


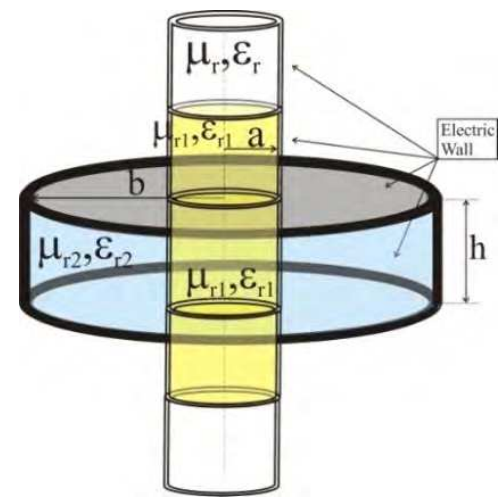

Fig. 6a. Circular cavity and the hole.

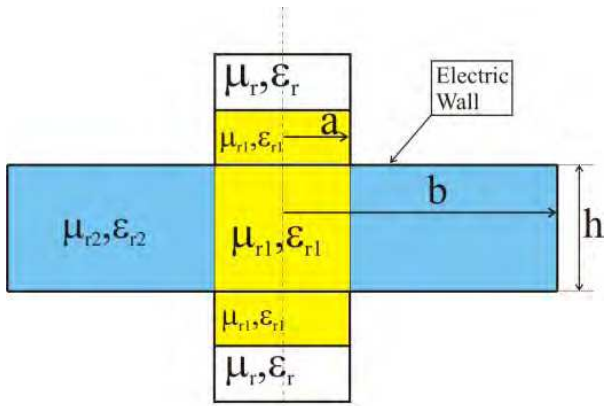

Fig. 6b. Circular cavity and the hole: section view.

As previously said, to completely analyze this structure we must divide it in simpler networks by a segmentation process. Figure 7a shows how this structure can be segmented. The segmentation consists of a division of the whole network into smaller ones, with canonical shapes in general, and then the analysis will be easier. So, the segmentation proposed in figure 7a divides the geometry into 3 different networks: one is a one-port network, in a circular ring shape with external radius $a$ and external radius $b$ and height $h$ where the one-port in put in the inner part $(r=a)$. This structure is shown in figure $7 \mathrm{~b}$. Another network is a two-port network that consists of a circular waveguide of radius $a$ and height $d$ whose ports are located in the top and in the bottom, as shown in figure 7c.

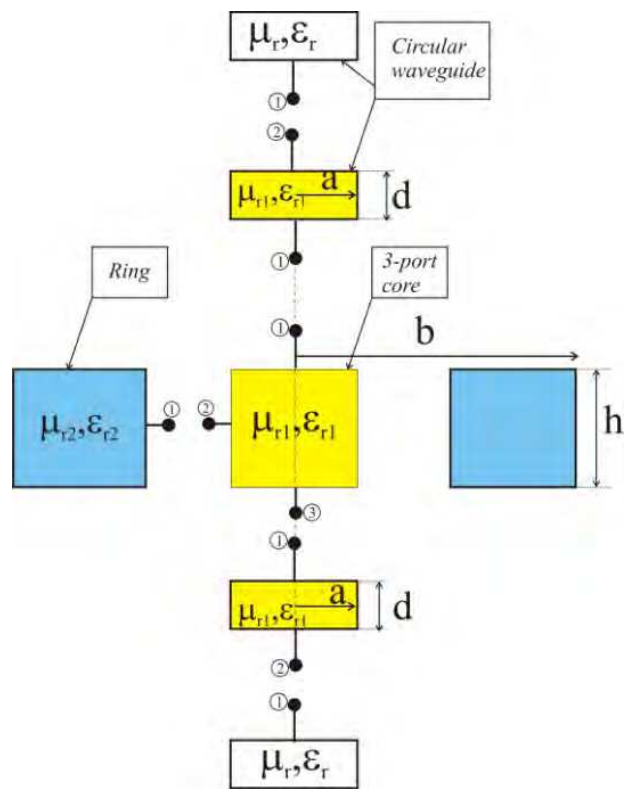

Fig. 7a. Circular cavity and the hole.

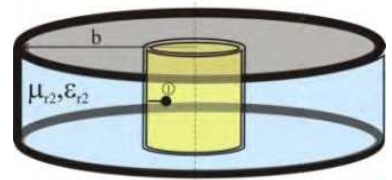

Fig. 7b. 1-port network: Ring.

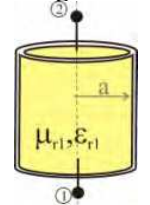

Fig. 7c. 2-port:

Waveguide

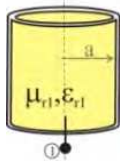

Fig. 7d. 1-port: Waveguide

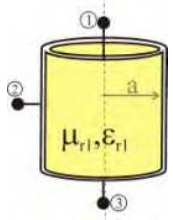

Fig. 7e. 3-port network: Core 
This network can be converted in a one-port network, located in the bottom, as shown in figure $7 \mathrm{~d}$, just considering that there is no reflection in port 2 . Finally a three-port network appears that consists of a circular tube of radius a and height $h$ with ports in the top and in the bottom, as before, but adding a port in the circular face, in $r=a$. This geometry is shown in figure 7e.

Then the original problem is reduced to 3 simpler problems: the circular ring, a circular waveguide and the 3-port guide.

\subsection{Analysis of a 1 port network}

Let's start calculating the Generalized Admittance Matrix (GAM) of the 1-port network shown in figure $7 \mathrm{~d}$. It is the simplest one and it will allow seeing the whole procedure.

Figure 8 shows the axis to be considered for this structure.

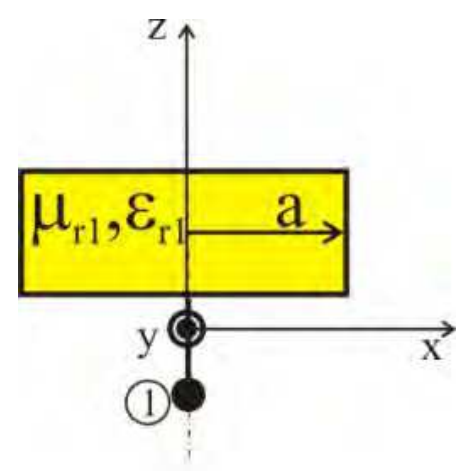

Fig. 8. One port network

The internal field in the circular waveguide is:

$$
\left\{\begin{array}{c}
E_{z}=\sum_{n=1}^{\infty} \frac{j \cdot k_{c n}^{2}}{\omega \cdot \varepsilon \cdot \mu} \cdot \mathrm{J}_{0}\left(k_{c n} \cdot r\right) \cdot\left(A_{n}^{+} \cdot e^{-\gamma_{n} \cdot z}+A_{n}^{-} \cdot e^{+\gamma_{n} \cdot z}\right) \\
\overrightarrow{\mathbf{E}}_{t}=E_{r} \cdot \hat{r}=\sum_{n=1}^{\infty} \frac{\gamma_{n} \cdot k_{c n}}{j \cdot \omega \cdot \varepsilon \cdot \mu} \cdot \mathrm{J}_{0}^{\prime}\left(k_{c m} \cdot r\right) \cdot\left(A_{n}^{+} \cdot e^{-\gamma_{n} \cdot z}-A_{n}^{-} \cdot e^{+\gamma_{n} \cdot z}\right) \cdot \hat{r} \\
\overrightarrow{\mathbf{H}}_{t}=H_{\varphi} \cdot \hat{\varphi}=\sum_{n=1}^{\infty} \frac{k_{c n}}{\mu} \cdot \mathrm{J}_{0}^{\prime}\left(k_{c n} \cdot r\right) \cdot\left(A_{n}^{+} \cdot e^{-\gamma_{n} \cdot z}+A_{n}^{-} \cdot e^{+\gamma_{n} \cdot z}\right) \cdot \hat{\varphi}
\end{array}\right.
$$

All the fields correspond to circular $\mathrm{TM}_{0 \mathrm{n}}$ modes (Balanis, 1989). As previously mentioned, only $\mathrm{TM}_{0 \mathrm{n}}$ modes are considered and, even there are a larger set of modes excited, like $\mathrm{TE}_{\mathrm{mn}}$ or $\mathrm{TM}_{\mathrm{mn}}$ in general, these modes are not coupled with the other ones. The resonant modes associated to these $\mathrm{TM}_{0 \mathrm{n}}$ modes are the $\mathrm{TM}_{0 \mathrm{np}}$ modes. Anyway, the proposed circuital method ca be used with any set of modes, so there is no loss of generality.

The propagation constant $\gamma_{n}$ is calculated from the cut-off wavenumbers $k_{c n}$ as (Balanis, 1989):

$$
k_{c n}^{2}=\omega^{2} \cdot \varepsilon \cdot \mu+\gamma_{n}^{2}=\omega^{2} \cdot \varepsilon_{0} \cdot \varepsilon_{r 1} \cdot \mu_{0} \cdot \mu_{r 1}+\gamma_{n}^{2}=k_{0}^{2} \cdot \varepsilon_{r 1} \cdot \mu_{r 1}+\gamma_{n}^{2}=\left(\frac{p_{0 n}}{a}\right)^{2}
$$


where $p_{0 n}$ are the zeros of the First Kind Bessel Functions of order $0\left(\mathrm{~J}_{0}\left(p_{0 n}\right)=0\right)$ and its value is obtained by focing the boundary conditions in the lateral faces (Balanis, 1989). The cut-off wavenumber is always a real number (Ramo, 1994) and teh criteria to select the sign of the propagation constant is (Baker-Jarvis et al., 1994):

$$
\gamma_{i}=\left\{\begin{array}{c}
j \cdot \sqrt{k^{2}-k_{c n}^{2}}=j \cdot \sqrt{k_{0}^{2} \cdot \varepsilon_{r 1} \cdot \mu_{r 1}-k_{c n}^{2}}, \operatorname{Re}(k) \geq k_{c n} \\
\sqrt{k_{c n}^{2}-k^{2}}=\sqrt{k_{c n}^{2}-k_{0}^{2} \cdot \varepsilon_{r 1} \cdot \mu_{r 1}}, \operatorname{Re}(k)<k_{c n}
\end{array}\right.
$$

Where the square root must be in the fist or in the forth quadrants.

Amplitudes $A_{n}^{+}$y $A_{n}^{-}$are, respectively, the forward and backward waves in the $\mathrm{z}$-axis direction, which is the propagation direction. In our particular case, because the waveguide is under cut-off, the backward waves are zero, so $A_{n}^{-}=0$.

To get the GAM we must incide with an electric field in port 1 . This electric field, as previously said, is written as a series expansions of base functions as:

$$
E_{i}^{(1)}=\sum_{m=1}^{\infty} \alpha_{m} \cdot e_{m}^{(1)}
$$

where the sleected base function for port 1 is:

$$
e_{m}^{(1)}=N_{m}^{(1)} \cdot J_{0}^{\prime}\left(\frac{p_{0 m}}{a} \cdot r\right)
$$

Term $N_{m}^{(1)}$ is a normalization term introduced in the modal analysis and we are going to use the proposed by Gentilli (Gentili, 1991). It must accomplish:

$$
I_{m q}=\int_{S} \mathbf{e}_{m}^{(1)}(r) \cdot \mathbf{e}_{q}^{(1)}(r) \cdot d S=\delta_{m n}= \begin{cases}0, & m \neq q \\ 1, & m=q\end{cases}
$$

Then the normalization term is:

$$
\begin{gathered}
\int_{r=0}^{a} \mathbf{e}_{m}^{(1)}(r) \cdot \mathbf{e}_{q}^{(1)}(r) \cdot r \cdot d r=\delta(m-q)=\int_{r=0}^{a}\left(N_{m}^{(1)}\right)^{2} \cdot \mathrm{J}_{1}^{2}\left(\frac{p_{0 m}}{a} \cdot r\right) \cdot r \cdot d r=\left(N_{m}^{(1)}\right)^{2} \cdot \frac{\left(a \cdot \mathrm{J}_{1}\left(p_{0 m}\right)\right)^{2}}{2}=1 \\
\Downarrow \\
N_{m}^{(1)}=\frac{\sqrt{2}}{a \cdot \mathrm{J}_{1}\left(p_{0 m}\right)}
\end{gathered}
$$

The incident field in port 1, equation (29), must equal to the electric field calculated from the inside of the structure, following equation(26). Then the forward amplitude is: 


$$
\begin{gathered}
E_{r}(z=0)=\sum_{n=1}^{\infty} \frac{\gamma_{n} \cdot k_{c n}}{j \cdot \omega \cdot \varepsilon \cdot \mu} \cdot \mathrm{J}_{0}^{\prime}\left(k_{c m} \cdot r\right) \cdot A_{n}^{+}=E_{i}=\sum_{m=1}^{\infty} \alpha_{m} \cdot e_{m}^{(1)}=\sum_{m=1}^{\infty} \alpha_{m} \cdot N_{m}^{(1)} \cdot J_{0}^{\prime}\left(\frac{p_{0 m}}{a} \cdot r\right) \\
A_{n}^{+}=\alpha_{m} \cdot N_{m}^{(1)} \cdot \frac{j \cdot \omega \cdot \varepsilon \cdot \mu}{\gamma_{n} \cdot k_{c n}} \cdot \delta(n-m)
\end{gathered}
$$

To get the GAM, we must obtain a relation between the electric and the magnetic fields in port 1 . The magnetic field in port 1 is, in the same way than the electric one:

$$
H^{(1)}=\sum_{q=1}^{\infty} c_{q} \cdot h_{q}^{(1)}
$$

where the base fucntion $h_{q}^{(1)}$ is the same than that used for electric field: $h_{q}^{(1)}=e_{q}^{(1)}$

And the GAM is defined as:

$$
\overrightarrow{\mathbf{h}}^{(1)}=\overline{\overline{\mathbf{Y}}}_{11} \cdot \overrightarrow{\mathbf{e}}^{(1)}
$$

where $\overrightarrow{\mathbf{e}}^{(1)}$ is a column vector with the electric field amplitudes at port 1 and $\overrightarrow{\mathbf{h}}^{(1)}$ is the column vector with the magnetic field amplitudes at port 1 :

$$
\overrightarrow{\mathbf{h}}^{(1)}=\left(\begin{array}{llll}
c_{1} & c_{2} & \ldots & c_{Q}
\end{array}\right)^{t} \quad ; \quad \overrightarrow{\mathbf{e}}^{(1)}=\left(\begin{array}{llll}
\alpha_{1} & \alpha_{2} & \ldots & \alpha_{M}
\end{array}\right)^{t}
$$

Then identifying the inner magnetic field in port 1 with the magnetic field from equation (34) we have:

$$
\begin{gathered}
H_{\varphi}(z=0)=\sum_{n=1}^{\infty} \frac{k_{c n}}{\mu} \cdot \mathrm{J}_{0}^{\prime}\left(k_{c n} \cdot r\right) \cdot A_{n}^{+}=\sum_{q=1}^{\infty} c_{q} \cdot h_{q}^{(1)}=\sum_{q=1}^{\infty} c_{q} \cdot N_{q}^{(1)} \cdot J_{0}^{\prime}\left(\frac{p_{0 q}}{a} \cdot r\right) \\
c_{q}=\alpha_{m} \cdot \frac{j \cdot \omega \cdot \varepsilon}{\gamma_{m}} \cdot \delta(q-m)
\end{gathered}
$$

Then the Generalized Admittance Matrix GAM is:

$$
\left.Y_{11}\right|_{q m}=\frac{j \cdot \omega \cdot \varepsilon}{\gamma_{m}} \cdot \delta(q-m)=\frac{j \cdot \omega \cdot \varepsilon_{0} \cdot \varepsilon_{r 1}}{\gamma_{m}} \cdot \delta(q-m)
$$

\subsection{Analysis of a 1-port ring structure}

This example will show how to calculate the GAM of the network shown in figure $7 \mathrm{~b}$. It is a 1 -port network, as before, but the port is located in the lateral.

Figure 9 shows the axis to be considered. 


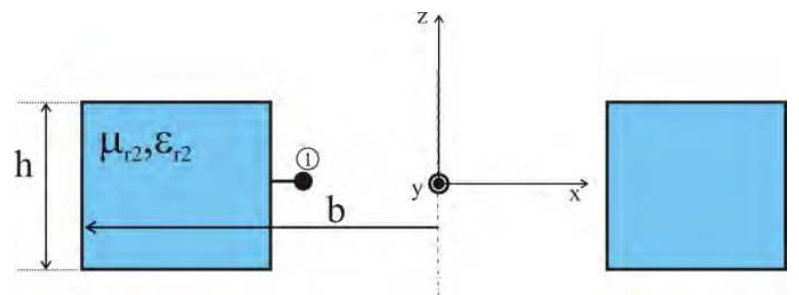

Fig. 9. One port network: ring

The fields inside are, as before:

$$
\left\{\begin{array}{c}
E_{z}=\sum_{n=1}^{\infty} \frac{j \cdot k_{c n}^{2}}{\omega \cdot \varepsilon \cdot \mu} \cdot f_{0 n}(r) \cdot\left(A_{n}^{+} \cdot e^{-\gamma_{n} \cdot z}+A_{n}^{-} \cdot e^{+\gamma_{n} \cdot z}\right) \\
\overrightarrow{\mathbf{E}}_{t}=E_{r} \cdot \hat{r}=\sum_{n=1}^{\infty} \frac{\gamma_{n} \cdot k_{c n}}{j \cdot \omega \cdot \varepsilon \cdot \mu} \cdot f_{0 n}^{\prime}(r) \cdot\left(A_{n}^{+} \cdot e^{-\gamma_{n} \cdot z}-A_{n}^{-} \cdot e^{+\gamma_{n} \cdot z}\right) \cdot \hat{r} \\
\overrightarrow{\mathbf{H}}_{t}=H_{\varphi} \cdot \hat{\varphi}=\sum_{n=1}^{\infty} \frac{k_{c n}}{\mu} \cdot f_{0 n}^{\prime}(r) \cdot\left(A_{n}^{+} \cdot e^{-\gamma_{n} \cdot z}+A_{n}^{-} \cdot e^{+\gamma_{n} \cdot z}\right) \cdot \hat{\varphi}
\end{array}\right.
$$

where functions $f_{0 n}(r)$ and $f_{0}^{\prime}(r)$ are:

$$
\left\{\begin{array}{l}
f_{0 n}(r)=\mathrm{J}_{0}\left(k_{c n} \cdot r\right)-\frac{\mathrm{J}_{0}\left(k_{c n} \cdot b\right)}{\mathrm{Y}_{0}\left(k_{c n} \cdot b\right)} \cdot \mathrm{Y}_{0}\left(k_{c n} \cdot r\right) \\
f_{0}^{\prime}(r)=\mathrm{J}_{0}^{\prime}\left(k_{c n} \cdot r\right)-\frac{\mathrm{J}_{0}\left(k_{c n} \cdot b\right)}{\mathrm{Y}_{0}\left(k_{c n} \cdot b\right)} \cdot \mathrm{Y}_{0}^{\prime}\left(k_{c n} \cdot r\right)
\end{array}\right.
$$

Again only $\mathrm{TM}_{0 \mathrm{n}}$ modes are considred.

The cut-off wavenumber $k_{c n}$ is calculated, in this case, as a fucntion of the propagation constant $\gamma_{n}$ as:

$$
k_{c n}^{2}=\omega^{2} \cdot \varepsilon \cdot \mu+\gamma_{n}^{2}=\omega^{2} \cdot \varepsilon_{0} \cdot \varepsilon_{r 2} \cdot \mu_{0} \cdot \mu_{r 2}+\gamma_{n}^{2}=k_{0}^{2} \cdot \varepsilon_{r 2} \cdot \mu_{r 2}+\gamma_{n}^{2}
$$

And the propagation constant is calculated from equations (39) by applying the boundary conditions in $r=b$, that is already accomplished, and in $z=0$ and $z=h$. Forcing these last two boundary conditions we have:

$$
\left\{\begin{aligned}
E_{r}(z=h / 2)=0 & \Rightarrow A_{n}^{-}=A_{n}^{-} \cdot e^{-2 \cdot \gamma_{n} \cdot h / 2}=A_{n}^{-} \cdot e^{-\gamma_{n} \cdot h} \\
E_{r}(z=-h / 2)=0 & \Rightarrow \sinh \left(\gamma_{n} \cdot h\right)=0 \Rightarrow \gamma_{n}=\frac{j \cdot n}{h}
\end{aligned}\right.
$$

Then the fields inside the ring are: 


$$
\left\{\begin{array}{c}
E_{z}=\sum_{n=1}^{\infty} \frac{j \cdot k_{c n}^{2}}{\omega \cdot \varepsilon \cdot \mu} \cdot f_{0 n}(r) \cdot 2 \cdot A_{n}^{+} \cdot e^{\gamma_{n} \cdot h / 2} \cdot \cosh \left(\gamma_{n} \cdot\left(z+\frac{h}{2}\right)\right) \\
\overrightarrow{\mathbf{E}}_{t}=E_{r} \cdot \hat{r}=\sum_{n=1}^{\infty} \frac{\gamma_{n} \cdot k_{c n}}{j \cdot \omega \cdot \varepsilon \cdot \mu} \cdot f_{0 n}^{\prime}(r) \cdot(-2) \cdot A_{n}^{+} \cdot e^{\gamma_{n} \cdot h / 2} \cdot \sinh \left(\gamma_{n} \cdot\left(z+\frac{h}{2}\right)\right) \cdot \hat{r} \\
\overrightarrow{\mathbf{H}}_{t}=H_{\varphi} \cdot \hat{\varphi}=\sum_{n=1}^{\infty} \frac{k_{c n}}{\mu} \cdot f_{0 n}^{\prime}(r) \cdot 2 \cdot A_{n}^{+} \cdot e^{\gamma_{n} \cdot h / 2} \cdot \cosh \left(\gamma_{n} \cdot\left(z+\frac{h}{2}\right)\right) \cdot \hat{\varphi}
\end{array}\right.
$$

But the electric field in port 1 must be a series expasions on a basis functions. In this case we will use:

$$
E_{i}^{(1)}=\sum_{m=1}^{\infty} \alpha_{m} \cdot \sin \left(2 \cdot \pi \cdot m \cdot \frac{z-h / 2}{h}\right)+\beta_{m} \cdot \cos \left(2 \cdot \pi \cdot m \cdot \frac{z-h / 2}{h}\right)
$$

where the basis functions are the trigonometric functions.

This incident electric field in port 1, equation (44), must be equal to the same electric field comuted from the field inside the structure, following equation (43). Then the amplitudes inside are:

$$
\left(\begin{array}{c}
\left.E_{z}(r=a)=\sum_{n=1}^{\infty} \frac{j \cdot k_{c n}^{2}}{\omega \cdot \varepsilon \cdot \mu} \cdot f_{0 n}(a) \cdot 2 \cdot A_{n}^{+} \cdot e^{\gamma_{n} \cdot h / 2} \cdot \cosh \left(\gamma_{n} \cdot\left(z+\frac{h}{2}\right)\right)=E_{i}^{(1)}=\right) \\
=\sum_{m=1}^{\infty} \alpha_{m} \cdot \sin \left(2 \cdot \pi \cdot m \cdot \frac{z-h / 2}{h}\right)+\beta_{m} \cdot \cos \left(2 \cdot \pi \cdot m \cdot \frac{z-h / 2}{h}\right) \\
A_{n}^{+}=\frac{\chi_{n}}{h} \cdot \frac{\omega \cdot \varepsilon \cdot \mu}{j \cdot k_{c n}^{2}} \cdot \frac{e^{-\gamma_{n} \cdot h / 2}}{2 \cdot f_{0 n}(a)} \cdot \sum_{m=1}^{\infty} \alpha_{m} \cdot I_{n m}^{(s 2)}+\beta_{m} \cdot I_{n m}^{(c 2)}
\end{array}\right)
$$

where $\chi_{n}, I_{n m}^{(s 2)}$ y $I_{n m}^{(c 2)}$ are $^{2}$ :

$$
\begin{gathered}
\chi_{n}= \begin{cases}1, & n=0 \\
2, & n \neq 0\end{cases} \\
I_{n m}^{(s 2)}=\int_{-h / 2}^{h / 2} \sin \left(2 \cdot \pi \cdot m \cdot \frac{z-h / 2}{h}\right) \cdot \cos \left(\pi \cdot n \cdot \frac{z+h / 2}{h}\right) \cdot d z= \\
=-2 \cdot m \cdot h \cdot \frac{\sin (n \cdot \pi / 2-m \cdot \pi)}{n+2 \cdot m} \cdot \operatorname{sinc}(n / 2-m) \\
I_{n m}^{(c 2)}=\int_{-h / 2}^{h / 2} \cos \left(2 \cdot \pi \cdot m \cdot \frac{z-h / 2}{h}\right) \cdot \cos \left(\pi \cdot n \cdot \frac{z+h / 2}{h}\right) \cdot d z= \\
=\frac{h}{\chi_{m}} \cdot \delta(n-2 \cdot m)
\end{gathered}
$$

2 Function sinc of $I_{n m}^{(s 2)}$ is defined as normal: $\operatorname{sinc}(x)=\frac{\sin (\pi \cdot x)}{\pi \cdot x}$ 
To get the GAM we must, again, obtain the relation between the electric and magnetic fields in port 1 . The magnetic field in port 1 is:

$$
H^{(1)}=\sum_{q=1}^{\infty} c_{q} \cdot \sin \left(2 \cdot \pi \cdot q \cdot \frac{z-h / 2}{h}\right)+d_{q} \cdot \cos \left(2 \cdot \pi \cdot q \cdot \frac{z-h / 2}{h}\right)
$$

And the GAM is defined as:

$$
\overrightarrow{\mathbf{h}}^{(1)}=\overline{\overline{\mathbf{Y}}}_{11} \cdot \overrightarrow{\mathbf{e}}^{(1)}
$$

where $\overrightarrow{\mathbf{e}}^{(1)}$ and $\overrightarrow{\mathbf{h}}^{(1)}$ are the column vectors with the electric and magnetic amplitudes in port 1:

$$
\overrightarrow{\mathbf{h}}^{(1)}=\left(\begin{array}{llllllllll}
c_{1} & c_{2} & \ldots & c_{Q} & d_{1} & d_{2} & \ldots & d_{Q}
\end{array}\right)^{t} \quad ; \quad \overrightarrow{\mathbf{e}}^{(1)}=\left(\begin{array}{lllllllll}
\alpha_{1} & \alpha_{2} & \ldots & \alpha_{M} & \beta_{1} & \beta_{2} & \ldots & \beta_{M}
\end{array}\right)^{t}
$$

Then, identifying the inner magnetic field in port 1, equation (43), and the magnetic field outside, from equation (47), and substituing $A_{n}^{+}$following (45):

$$
\begin{gathered}
\left(\begin{array}{c}
H_{\varphi}(r=a)=\sum_{n=1}^{\infty} \frac{k_{c n}}{\mu} \cdot f_{0 n}^{\prime}(a) \cdot 2 \cdot A_{n}^{+} \cdot e^{\gamma_{n} \cdot h / 2} \cdot \cosh \left(\gamma_{n} \cdot\left(z+\frac{h}{2}\right)\right)= \\
=\sum_{q=1}^{\infty} c_{q} \cdot \sin \left(2 \cdot \pi \cdot q \cdot \frac{z-h / 2}{h}\right)+d_{q} \cdot \cos \left(2 \cdot \pi \cdot q \cdot \frac{z-h / 2}{h}\right)
\end{array}\right) \\
\left\{\begin{array}{c}
\Downarrow \\
c_{q}=\frac{2 \cdot\left(\chi_{q}-1\right)}{h} \cdot \sum_{n=1}^{\infty} \frac{\chi_{n}}{h} \cdot \frac{\omega \cdot \varepsilon}{j \cdot k_{c n}} \cdot \frac{f_{0 n}^{\prime}(a)}{f_{0 n}(a)} \cdot I_{n q}^{(s 2)} \cdot \sum_{m=1}^{\infty} \alpha_{m} \cdot I_{n m}^{(s 2)}+\beta_{m} \cdot I_{n m}^{(c 2)} \\
d_{q}=\frac{\chi_{q}}{h} \cdot \sum_{n=1}^{\infty} \frac{\chi_{n}}{h} \cdot \frac{\omega \cdot \varepsilon}{j \cdot k_{c n}} \cdot \frac{f_{0 n}^{\prime}(a)}{f_{0 n}(a)} \cdot I_{n q}^{(c 2)} \cdot \sum_{m=1}^{\infty} \alpha_{m} \cdot I_{n m}^{(s 2)}+\beta_{m} \cdot I_{n m}^{(c 2)}
\end{array}\right.
\end{gathered}
$$

On the other way, the GAM is:

$$
\overrightarrow{\mathbf{h}}^{(1)}=\left(\begin{array}{l}
\overrightarrow{\mathbf{c}} \\
\overrightarrow{\mathbf{d}}
\end{array}\right)=\overline{\overline{\mathbf{Y}}}_{11} \cdot \overrightarrow{\mathbf{e}}^{(1)}=\left(\begin{array}{ll}
\overline{\overline{\mathbf{Y}}}_{11}^{(s s)} & \overline{\overline{\mathbf{Y}}}_{11}^{(s c)} \\
\overline{\overline{\mathbf{Y}}}_{11} & \overline{\overline{\mathbf{Y}}}_{11}^{(c c)}
\end{array}\right) \cdot\left(\begin{array}{l}
\overrightarrow{\mathbf{\alpha}} \\
\overrightarrow{\boldsymbol{\beta}}
\end{array}\right)
$$

where each submatrix $\overline{\overline{\mathbf{Y}}}_{11}^{(a b)}$ is:

$$
\begin{aligned}
\left.\overline{\overline{\mathbf{Y}}}_{11}^{(s s)}\right|_{q m} & =\frac{2 \cdot\left(\chi_{q}-1\right)}{h} \cdot \sum_{n=1}^{\infty} \frac{\chi_{n}}{h} \cdot \frac{\omega \cdot \varepsilon}{j \cdot k_{c n}} \cdot \frac{f_{0 n}^{\prime}(a)}{f_{0 n}(a)} \cdot I_{n q}^{(s 2)} \cdot I_{n m}^{(s 2)} \\
\left.\overline{\overline{\mathbf{Y}}}_{11}^{(s c)}\right|_{q m} & =\frac{2 \cdot\left(\chi_{q}-1\right)}{h} \cdot \sum_{n=1}^{\infty} \frac{\chi_{n}}{h} \cdot \frac{\omega \cdot \varepsilon}{j \cdot k_{c n}} \cdot \frac{f_{0 n}^{\prime}(a)}{f_{0 n}(a)} \cdot I_{n q}^{(s 2)} \cdot I_{n m}^{(c 2)} \\
& \left.\overline{\overline{\mathbf{Y}}}_{11}^{(c s)}\right|_{q m}=\frac{\chi_{q}}{h} \cdot \sum_{n=1}^{\infty} \frac{\chi_{n}}{h} \cdot \frac{\omega \cdot \varepsilon}{j \cdot k_{c n}} \cdot \frac{f_{0 n}^{\prime}(a)}{f_{0 n}(a)} \cdot I_{n q}^{(c 2)} \cdot I_{n m}^{(s 2)}
\end{aligned}
$$




$$
\left.\overline{\overline{\mathbf{Y}}}_{11}^{(c c)}\right|_{q m}=\frac{\chi_{q}}{h} \cdot \sum_{n=1}^{\infty} \frac{\chi_{n}}{h} \cdot \frac{\omega \cdot \varepsilon}{j \cdot k_{c n}} \cdot \frac{f_{0 n}^{\prime}(a)}{f_{0 n}(a)} \cdot I_{n q}^{(c 2)} \cdot I_{n m}^{(c 2)}
$$

\subsection{Analysis of a 3-port network}

Now let us calculate the GAM of the 3-port network depicted in figure 7e. This network has the two before cases, because it has ports in the top, in the bottom and in the lateral side.

Figure 10 shows the axis to be used in this 3-port network.

Fig. 10. Three port network: core

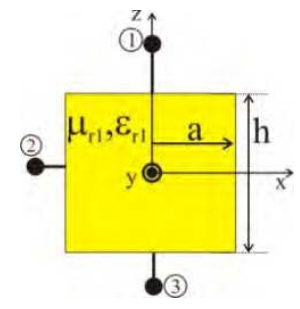

Note that now the GAM is $3 \times 3$ dimension matrix:

$$
\overrightarrow{\mathbf{h}}=\overline{\overline{\mathbf{Y}}} \cdot \overrightarrow{\mathbf{e}} \Rightarrow\left(\begin{array}{l}
\overrightarrow{\mathbf{h}}^{(1)} \\
\overrightarrow{\mathbf{h}}^{(2)} \\
\overrightarrow{\mathbf{h}}^{(3)}
\end{array}\right)=\left(\begin{array}{lll}
\overline{\overline{\mathbf{Y}}}_{11} & \overline{\overline{\mathbf{Y}}}_{12} & \overline{\overline{\mathbf{Y}}}_{13} \\
\overline{\overline{\mathbf{Y}}}_{21} & \overline{\overline{\mathbf{Y}}}_{22} & \overline{\overline{\mathbf{Y}}}_{23} \\
\overline{\overline{\mathbf{Y}}}_{31} & \overline{\overline{\mathbf{Y}}}_{32} & \overline{\overline{\mathbf{Y}}}_{33}
\end{array}\right) \cdot\left(\begin{array}{c}
\overrightarrow{\mathbf{e}}^{(1)} \\
\overrightarrow{\mathbf{e}}^{(2)} \\
\overrightarrow{\mathbf{e}}^{(3)}
\end{array}\right)
$$

To analyse this multiport structure we must go by columns, in such a way that all the ports are short-circuited except port $j$, where $j=(1,2,3)$. So, $\left.\overrightarrow{\mathbf{e}}^{(i)}\right|_{i \neq j}=0$, and then the GAM elements are computed as (the $j$ column in this case):

$$
\overrightarrow{\mathbf{h}}^{(k)}=\left.\overline{\overline{\mathbf{Y}}}_{k j} \cdot \overrightarrow{\mathbf{e}}^{(j)}\right|_{k=1,2,3}
$$

\subsubsection{Parameters $\overline{\overline{\mathbf{Y}}}_{i 1}$}

To compute this column we must short-circuit ports 2 and 3 . Then in $z=-h / 2$ and in $r=a$ we have electric walls.

In general, the fields inside the structure will be, considering only $\mathrm{TM}_{0 \mathrm{n}}$ modes:

$$
\left\{\begin{array}{c}
E_{z}=\sum_{n=1}^{\infty} \frac{j \cdot k_{c n}^{2}}{\omega \cdot \varepsilon \cdot \mu} \cdot \mathrm{J}_{0}\left(k_{c n} \cdot r\right) \cdot\left(A_{n}^{+} \cdot e^{-\gamma_{n} \cdot z}+A_{n}^{-} \cdot e^{+\gamma_{n} \cdot z}\right) \\
\mathbf{E}_{t}=E_{r} \cdot \hat{r}=\sum_{n=1}^{\infty} \frac{\gamma_{n} \cdot k_{c n}}{j \cdot \omega \cdot \varepsilon \cdot \mu} \cdot \mathrm{J}_{0}^{\prime}\left(k_{c n} \cdot r\right) \cdot\left(A_{n}^{+} \cdot e^{-\gamma_{n} \cdot z}-A_{n}^{-} \cdot e^{+\gamma_{n} \cdot z}\right) \cdot \hat{r} \\
\overrightarrow{\mathbf{H}}_{t}=H_{\varphi} \cdot \hat{\varphi}=\sum_{n=1}^{\infty} \frac{k_{c n}}{\mu} \cdot \mathrm{J}_{0}^{\prime}\left(k_{c n} \cdot r\right) \cdot\left(A_{n}^{+} \cdot e^{-\gamma_{n} \cdot z}+A_{n}^{-} \cdot e^{+\gamma_{n} \cdot z}\right) \cdot \hat{\varphi}
\end{array}\right.
$$


where the Second Kind Bessel Functions $Y_{0}\left(k_{c n} \cdot r\right)$ disappear because they are singular in the origin, and where the cut-off wavenumber $k_{c n}$ and the propagation constant $\gamma_{n}$ are related as:

$$
k_{c n}^{2}=\omega^{2} \cdot \varepsilon \cdot \mu+\gamma_{n}^{2}=\omega^{2} \cdot \varepsilon_{0} \cdot \varepsilon_{r 1} \cdot \mu_{0} \cdot \mu_{r 1}+\gamma_{n}^{2}=k_{0}^{2} \cdot \varepsilon_{r 1} \cdot \mu_{r 1}+\gamma_{n}^{2}
$$

The boundary condition are then:

$$
\left\{\begin{array}{c}
E_{r}(z=h / 2)=0 \Rightarrow A_{n}^{-}=A_{n}^{-} \cdot e^{-2 \cdot \gamma_{n} \cdot h / 2}=A_{n}^{-} \cdot e^{-\gamma_{n} \cdot h} \\
E_{z}(r=a)=0 \Rightarrow k_{c n}=\frac{p_{0 n}}{a}
\end{array}\right.
$$

And the electromagnetic fields inside are:

$$
\left\{\begin{array}{c}
E_{z}=\sum_{n=1}^{\infty} \frac{j \cdot k_{c n}^{2}}{\omega \cdot \varepsilon \cdot \mu} \cdot \mathrm{J}_{0}\left(k_{c n} \cdot r\right) \cdot 2 \cdot A_{n}^{+} \cdot e^{\gamma_{n} \cdot h / 2} \cdot \cosh \left(\gamma_{n} \cdot\left(z+\frac{h}{2}\right)\right) \\
\overrightarrow{\mathbf{E}}_{t}=E_{r} \cdot \hat{r}=\sum_{n=1}^{\infty} \frac{-\gamma_{n} \cdot k_{c n}}{j \cdot \omega \cdot \varepsilon \cdot \mu} \cdot \mathrm{J}_{0}^{\prime}\left(k_{c n} \cdot r\right) \cdot 2 \cdot A_{n}^{+} \cdot e^{\gamma_{n} \cdot h / 2} \cdot \sinh \left(\gamma_{n} \cdot\left(z+\frac{h}{2}\right)\right) \cdot \hat{r} \\
\overrightarrow{\mathbf{H}}_{t}=H_{\varphi} \cdot \hat{\varphi}=\sum_{n=1}^{\infty} \frac{k_{c n}}{\mu} \cdot \mathrm{J}_{0}^{\prime}\left(k_{c n} \cdot r\right) \cdot 2 \cdot A_{n}^{+} \cdot e^{\gamma_{n} \cdot h / 2} \cdot \cosh \left(\gamma_{n} \cdot\left(z+\frac{h}{2}\right)\right) \cdot \hat{\varphi}
\end{array}\right.
$$

Identifying now the tangential incident field in port 1 with the ineternal field in the plane, as made in equation (33), and using the same series expansion for the incident electric field than before, as in equation (29), we have:

$$
\begin{gathered}
\sum_{n=1}^{\infty} \frac{\gamma_{n} \cdot k_{c n} \cdot 2}{j \cdot \omega \cdot \varepsilon \cdot \mu} \cdot \mathrm{J}_{1}\left(k_{c m} \cdot r\right) \cdot A_{n}^{+} \cdot e^{\gamma_{n} \cdot h / 2} \cdot \sinh \left(\gamma_{n} \cdot h\right)=\sum_{m=1}^{\infty} \alpha_{m} \cdot N_{m}^{(1)} \cdot J_{0}^{\prime}\left(\frac{p_{0 m}}{a} \cdot r\right) \\
\Downarrow \\
A_{n}^{+}=\alpha_{m} \cdot N_{m}^{(1)} \cdot \frac{j \cdot \omega \cdot \varepsilon \cdot \mu}{\gamma_{n} \cdot k_{c n}} \cdot \frac{e^{\gamma_{n} \cdot h / 2}}{-2 \cdot \sinh \left(\gamma_{n} \cdot h\right)} \cdot \delta(n-m)
\end{gathered}
$$

Following this, parameter $\overline{\overline{\mathbf{Y}}}_{11}$ must accomplish: $\overrightarrow{\mathbf{h}}^{(1)}=\overline{\overline{\mathbf{Y}}}_{11} \cdot \overrightarrow{\mathbf{e}}^{(1)}$. The magnetic field will be, in the same way than in point 3.4 and showed in equation (34):

$$
\left.\begin{array}{rl}
\left(H_{\varphi}\left(z=\frac{h}{2}\right)\right. & =\sum_{n=1}^{\infty} \frac{k_{c n}}{\mu} \cdot \mathrm{J}_{0}^{\prime}\left(k_{c n} \cdot r\right) \cdot 2 \cdot A_{n}^{+} \cdot e^{\gamma_{n} \cdot h / 2} \cdot \cosh \left(\gamma_{n} \cdot h\right)= \\
& =\sum_{q=1}^{\infty} c_{q} \cdot h_{q}^{(1)}=\sum_{q=1}^{\infty} c_{q} \cdot N_{q}^{(1)} \cdot J_{0}^{\prime}\left(\frac{p_{0 q}}{a} \cdot r\right)
\end{array}\right)
$$


Then the GAM is:

$$
\left.Y_{11}\right|_{q m}=\frac{-j \cdot \omega \cdot \varepsilon_{0} \cdot \varepsilon_{r 1}}{\gamma_{m}} \cdot \frac{1}{\tanh \left(\gamma_{n} \cdot h\right)} \cdot \delta(q-m)
$$

In the same way, parameter $\overline{\overline{\mathbf{Y}}}_{31}$ is that who accomplishes: $\overrightarrow{\mathbf{h}}^{(3)}=\overline{\overline{\mathbf{Y}}}_{31} \cdot \overrightarrow{\mathbf{e}}^{(1)}$. The magnetic field is, as shown in point 3.4 and in equation (34), and equating as before:

$$
\left.Y_{31}\right|_{q m}=\frac{-j \cdot \omega \cdot \varepsilon_{0} \cdot \varepsilon_{r 1}}{\gamma_{m}} \cdot \frac{1}{\sinh \left(\gamma_{n} \cdot h\right)} \cdot \delta(q-m)
$$

And parameter $\overline{\overline{\mathbf{Y}}}_{21}$, who accomplishes $\overrightarrow{\mathbf{h}}^{(2)}=\overline{\overline{\mathbf{Y}}}_{21} \cdot \overrightarrow{\mathbf{e}}^{(1)}$, is calculated in a similar way, remebering that the tangential magnetic field in port 2 is expanded in a series as shown in (47). Identifying the magnetic fields, we have, after replacing $A_{n}^{+}$with the value obtained in equation (59):

$$
\begin{aligned}
& \left(\begin{array}{c}
H_{\varphi}(r=a)=\sum_{n=1}^{\infty} \frac{k_{c n}}{\mu} \cdot \mathrm{J}_{0}^{\prime}\left(k_{c n} \cdot a\right) \cdot 2 \cdot A_{n}^{+} \cdot e^{\gamma_{n} \cdot h / 2} \cdot \cosh \left(\gamma_{n} \cdot\left(z+\frac{h}{2}\right)\right)= \\
=\sum_{q=1}^{\infty} c_{q} \cdot \sin \left(2 \cdot \pi \cdot q \cdot \frac{z-h / 2}{h}\right)+d_{q} \cdot \cos \left(2 \cdot \pi \cdot q \cdot \frac{z-h / 2}{h}\right)
\end{array}\right) \\
& \left\{\begin{array}{c}
c_{q}=\frac{2 \cdot\left(\chi_{q}-1\right)}{h} \cdot \sum_{m=1}^{\infty} \alpha_{m} \cdot \mathrm{J}_{0}^{\prime}\left(p_{0 m}\right) \cdot \frac{\omega \cdot \varepsilon}{j \cdot \gamma_{m}} \cdot \frac{N_{m}^{(1)}}{\cosh \left(\gamma_{m} \cdot h\right)} \cdot I_{q m}^{(s)} \\
d_{q}=\frac{\chi_{q}}{h} \cdot \sum_{n=1}^{\infty} \alpha_{m} \cdot \mathrm{J}_{0}^{\prime}\left(p_{0 m}\right) \cdot \frac{\omega \cdot \varepsilon}{j \cdot \gamma_{m}} \cdot \frac{N_{m}^{(1)}}{\cosh \left(\gamma_{m} \cdot h\right)} \cdot I_{q m}^{(c)}
\end{array}\right.
\end{aligned}
$$

where $\chi_{n}$ and $\operatorname{sinc}(x)$ have been defined before and $I_{q m}^{(s, c)}$ are 3 (more general than (46)):

$$
\begin{gathered}
I_{q m}^{(s)}=\int_{-h / 2}^{h / 2} \sin \left(2 \cdot \pi \cdot q \cdot \frac{z-h / 2}{h}\right) \cdot \cosh \left(\gamma_{m} \cdot(z+h / 2)\right) \cdot d z= \\
=\frac{h \cdot \pi \cdot q}{j \cdot \pi \cdot q+\gamma_{m} \cdot h} \cdot \sinh \left(\gamma_{m} \cdot h / 2-j \cdot \pi \cdot q\right) \cdot \sinh \left(\gamma_{m} \cdot h / 2-j \cdot \pi \cdot q\right)= \\
=-\int_{-h / 2}^{h / 2} \sin \left(2 \cdot \pi \cdot q \cdot \frac{z-h / 2}{h}\right) \cdot \cosh \left(\gamma_{m} \cdot(z-h / 2)\right) \cdot d z
\end{gathered}
$$

${ }^{3}$ Function $\sinh c$ of $I_{q m}^{(s, c)}$ is: $\sinh c(x)=\frac{\sinh (x)}{x}$ (note the difference with $\operatorname{sinc}$ ) 


$$
\begin{aligned}
I_{q m}^{(c)}=\int_{-h / 2}^{h / 2} \cos \left(2 \cdot \pi \cdot q \cdot \frac{z-h / 2}{h}\right) \cdot \cosh \left(\gamma_{m} \cdot(z+h / 2)\right) \cdot d z= \\
=h^{2} \cdot \gamma_{m} \cdot \frac{\sinh \left(\gamma_{m} \cdot h-j \cdot 2 \cdot \pi \cdot q\right)}{\gamma_{m} \cdot h+j \cdot 2 \cdot \pi \cdot q}= \\
=\int_{-h / 2}^{h / 2} \cos \left(2 \cdot \pi \cdot q \cdot \frac{z-h / 2}{h}\right) \cdot \cosh \left(\gamma_{m} \cdot(z-h / 2)\right) \cdot d z
\end{aligned}
$$

Then parameter $\overline{\overline{\mathbf{Y}}}_{21}$, defined as $\overrightarrow{\mathbf{h}}^{(2)}=\left(\begin{array}{c}\overrightarrow{\mathbf{c}} \\ \overrightarrow{\mathbf{d}}\end{array}\right)=\overline{\overline{\mathbf{Y}}}_{21} \cdot \overrightarrow{\mathbf{e}}^{(1)}=\left(\begin{array}{c}\overline{\overline{\mathbf{Y}}}_{21}^{(s)} \\ \overline{\overline{\mathbf{Y}}}_{21}^{(c)}\end{array}\right) \cdot \overrightarrow{\boldsymbol{\alpha}}$, is:

where each submatrix $\overline{\overline{\mathbf{Y}}}_{21}^{(s, c)}$ is:

$$
\begin{array}{r}
\left.\overline{\overline{\mathbf{Y}}}_{21}^{(s)}\right|_{q m}=\frac{2 \cdot\left(\chi_{q}-1\right)}{h} \cdot \mathrm{J}_{0}^{\prime}\left(p_{0 m}\right) \cdot \frac{\omega \cdot \varepsilon}{\gamma_{m}} \cdot \frac{N_{m}^{(1)}}{\sinh \left(\gamma_{m} \cdot h\right)} \cdot I_{q m}^{(s)} \\
\left.\overline{\overline{\mathbf{Y}}}_{21}^{(c)}\right|_{q m}=\frac{\chi_{q}}{h} \cdot \mathrm{J}_{0}^{\prime}\left(p_{0 m}\right) \cdot \frac{\omega \cdot \varepsilon}{\gamma_{m}} \cdot \frac{N_{m}^{(1)}}{\sinh \left(\gamma_{m} \cdot h\right)} \cdot I_{q m}^{(c)}
\end{array}
$$

\subsubsection{Parameters $\overline{\overline{\mathbf{Y}}}_{i 2}$}

For this column number 2, we must put short-circuits in ports 1 and 3 . Then in $z=-h / 2$ and in $z=+h / 2$ we have electric walls.

In general, the inner electromagnetic fields are, as before for $\mathrm{TM}_{0 \mathrm{n}}$ modes:

$$
\left\{\begin{array}{c}
E_{z}=\sum_{n=1}^{\infty} \frac{j \cdot k_{c n}^{2}}{\omega \cdot \varepsilon \cdot \mu} \cdot \mathrm{J}_{0}\left(k_{c n} \cdot r\right) \cdot\left(A_{n}^{+} \cdot e^{-\gamma_{n} \cdot z}+A_{n}^{-} \cdot e^{+\gamma_{n} \cdot z}\right) \\
\overrightarrow{\mathbf{E}}_{t}=E_{r} \cdot \hat{r}=\sum_{n=1}^{\infty} \frac{\gamma_{n} \cdot k_{c n}}{j \cdot \omega \cdot \varepsilon \cdot \mu} \cdot \mathrm{J}_{0}^{\prime}\left(k_{c n} \cdot r\right) \cdot\left(A_{n}^{+} \cdot e^{-\gamma_{n} \cdot z}-A_{n}^{-} \cdot e^{+\gamma_{n} \cdot z}\right) \cdot \hat{r} \\
\overrightarrow{\mathbf{H}}_{t}=H_{\varphi} \cdot \hat{\varphi}=\sum_{n=1}^{\infty} \frac{k_{c n}}{\mu} \cdot \mathrm{J}_{0}^{\prime}\left(k_{c n} \cdot r\right) \cdot\left(A_{n}^{+} \cdot e^{-\gamma_{n} \cdot z}+A_{n}^{-} \cdot e^{+\gamma_{n} \cdot z}\right) \cdot \hat{\varphi}
\end{array}\right.
$$

where the cut-off wavenumber $k_{c n}$ and the propagation constant $\gamma_{n}$ are related as:

$$
k_{c n}^{2}=\omega^{2} \cdot \varepsilon \cdot \mu+\gamma_{n}^{2}=\omega^{2} \cdot \varepsilon_{0} \cdot \varepsilon_{r 1} \cdot \mu_{0} \cdot \mu_{r 1}+\gamma_{n}^{2}=k_{0}^{2} \cdot \varepsilon_{r 1} \cdot \mu_{r 1}+\gamma_{n}^{2}
$$

Imposing the boundary conditions we have:

$$
\left\{\begin{array}{c}
E_{z}(z=h / 2)=0 \Rightarrow A_{n}^{-}=A_{n}^{-} \cdot e^{-2 \cdot \gamma_{n} \cdot h / 2}=A_{n}^{-} \cdot e^{-\gamma_{n} \cdot h} \\
E_{z}(z=-h / 2)=0 \Rightarrow \gamma_{n}=j \cdot \beta_{n}=j \cdot \frac{n \cdot \pi}{h}
\end{array}\right.
$$

And the inner fields: 


$$
\left\{\begin{array}{c}
E_{z}=\sum_{n=1}^{\infty} \frac{j \cdot k_{c n}^{2}}{\omega \cdot \varepsilon \cdot \mu} \cdot \mathrm{J}_{0}\left(k_{c n} \cdot r\right) \cdot 2 \cdot A_{n}^{+} \cdot e^{\gamma_{n} \cdot h / 2} \cdot \cosh \left(\gamma_{n} \cdot\left(z+\frac{h}{2}\right)\right) \\
\overrightarrow{\mathbf{E}}_{t}=E_{r} \cdot \hat{r}=\sum_{n=1}^{\infty} \frac{-\gamma_{n} \cdot k_{c n}}{j \cdot \omega \cdot \varepsilon \cdot \mu} \cdot \mathrm{J}_{0}^{\prime}\left(k_{c n} \cdot r\right) \cdot 2 \cdot A_{n}^{+} \cdot e^{\gamma_{n} \cdot h / 2} \cdot \sinh \left(\gamma_{n} \cdot\left(z+\frac{h}{2}\right)\right) \cdot \hat{r} \\
\overrightarrow{\mathbf{H}}_{t}=H_{\varphi} \cdot \hat{\varphi}=\sum_{n=1}^{\infty} \frac{k_{c n}}{\mu} \cdot \mathrm{J}_{0}^{\prime}\left(k_{c n} \cdot r\right) \cdot 2 \cdot A_{n}^{+} \cdot e^{\gamma_{n} \cdot h / 2} \cdot \cosh \left(\gamma_{n} \cdot\left(z+\frac{h}{2}\right)\right) \cdot \hat{\varphi}
\end{array}\right.
$$

Identifying the incident tangential electric field in port 2 with the inner electric field, from equation (45), and using the same series expansion in the incident field than in equation (44):

$$
\begin{gathered}
\left(\begin{array}{c}
\sum_{n=1}^{\infty} \frac{j \cdot k_{c n}^{2}}{\omega \cdot \varepsilon \cdot \mu} \cdot \mathrm{J}_{0}\left(k_{c n} \cdot a\right) \cdot 2 \cdot A_{n}^{+} \cdot e^{\gamma_{n} \cdot h / 2} \cdot \cos \left(\frac{n \cdot \pi}{h} \cdot\left(z+\frac{h}{2}\right)\right)= \\
=\sum_{m=1}^{\infty} \alpha_{m} \cdot \sin \left(2 \cdot \pi \cdot m \cdot \frac{z-h / 2}{h}\right)+\beta_{m} \cdot \cos \left(2 \cdot \pi \cdot m \cdot \frac{z-h / 2}{h}\right)
\end{array}\right) \\
A_{n}^{+}=\frac{\chi_{n}}{h} \cdot \frac{\omega \cdot \varepsilon \cdot \mu}{j \cdot k_{c n}^{2}} \cdot \frac{e^{-\gamma_{n} \cdot h / 2}}{2 \cdot \mathrm{J}_{0}\left(k_{c n} \cdot a\right)} \cdot \sum_{m=1}^{\infty} \alpha_{m} \cdot I_{n m}^{(s 2)}+\beta_{m} \cdot I_{n m}^{(c 2)}
\end{gathered}
$$

Then, parameters $\overline{\overline{\mathbf{Y}}}_{12}, \overline{\overline{\mathbf{Y}}}_{22}$ and $\overline{\overline{\mathbf{Y}}}_{32}$ are calculated easily using the same procedure than before.

\subsubsection{Parameters $\overline{\overline{\mathbf{Y}}}_{i 3}$}

Finally, parameters of the $3^{\text {rd }}$ column are calulated exactly in the same way than parameters $\overline{\overline{\mathbf{Y}}}_{i 1}$.

\section{Simulations and measurements}

Once all the GAM in figure 7a are obtained, it is quite easy and fast to analyse the geometries proposed in figures $6 \mathrm{a}$ and $6 \mathrm{~b}$. To do this is enough to apply the theory and equations proposed in point

2.3 .

Those expressions allow interconnecting different networks just knowing the corresponding multimodal matrices GAM, GIM or GSM.

At this point is important to note that each multimodal matrix can use only one mode. Then they are called monomode expressions. These monomode expressions are less accurate but they are a really good seed for a more accurate result.

The monomode expressions were used and presented by the authors in (Penaranda-Foix et al., 2007b). If we look at the cavity shown in figure $7 \mathrm{a}$, to get the resonant frequency is reduced to the simplest resonant condition, shown as an example in (Penaranda-Foix et al., 
2009). When only one mode is considered, the monomode analysis takes us to the expression (10) of (Penaranda-Foix et al., 2007b):

$$
1+\frac{j \cdot k_{0} \cdot \varepsilon_{r 1}}{a \cdot h \cdot \beta_{z 1}^{3}} \cdot \frac{1}{\frac{f_{1}(a)}{f_{0}(a)}-\sqrt{\frac{\varepsilon_{r 1}}{\mu_{r 1}}} \cdot \frac{\mathrm{J}_{1}\left(k_{0} \cdot \sqrt{\varepsilon_{r 1} \cdot \mu_{r 1}} \cdot a\right)}{\mathrm{J}_{0}\left(k_{0} \cdot \sqrt{\varepsilon_{r 1} \cdot \mu_{r 1}} \cdot a\right)}}=0
$$

where all the permitivities and permeabilities but those of the central material to be measured, are air, and where:

$$
\begin{gathered}
f_{n}(a)=\frac{\mathrm{J}_{n}\left(k_{0} \cdot a\right)-\frac{\mathrm{J}_{0}\left(k_{0} \cdot b\right)}{\mathrm{Y}_{0}\left(k_{0} \cdot b\right)} \cdot \mathrm{Y}_{n}\left(k_{0} \cdot b\right)}{\mathrm{J}_{0}\left(k_{0} \cdot a\right)-\frac{\mathrm{J}_{0}\left(k_{0} \cdot b\right)}{\mathrm{Y}_{0}\left(k_{0} \cdot b\right)} \cdot \mathrm{Y}_{0}\left(k_{0} \cdot b\right)} \\
\beta_{z 1}^{3}=k_{0}^{2} \cdot \varepsilon_{r 1} \cdot \mu_{r 1}-\left(p_{01} / a\right)^{2}
\end{gathered}
$$

To obtain equation (71) the insertion hole is only in the top, having a short-circuito in the bottom. That menas that in port 3 a short-circuited has been placed. Using the same procedure, a similar expression can be achieved with an insertion hole in the top and in the bottom. When no insertion hole is placed, the analytical analitycal procedure can be used (see Balanis, 1989)). Using the monomode equations previously determined, we arrive to the equation (8) of (Penaranda-Foix et al., 2007b):

$$
\frac{f_{1}(a)}{f_{0}(a)}-\sqrt{\frac{\varepsilon_{r 1}}{\mu_{r 1}}} \cdot \frac{\mathrm{J}_{1}\left(k_{0} \cdot \sqrt{\varepsilon_{r 1} \cdot \mu_{r 1}} \cdot a\right)}{\mathrm{J}_{0}\left(k_{0} \cdot \sqrt{\varepsilon_{r 1} \cdot \mu_{r 1}} \cdot a\right)}=0
$$

Of course, this equation is the same that that obtained by Balanis. The result is normal, because the Balanis procedure consists of a monomode analysis.

The resonant frequencies obtained with and without insertion hole are different. This frequency deviation can be really important when measuring large permittivities or large aspect ratio cavities. It was 1960 when Estin (Estin \& Bussey, 1960) published a first aproximate expression to estimate this error. It was a linear equation and it did not take into account the saturation effect described by the monomode procedure. It appears at large permittivities and at large aspect rates. Then in 2007 Penaranda-Foix (Penaranda-Foix et al., 2007c) proposed an alternative exponential equation obtained from the exact value and (71):

$$
\frac{\delta f}{f_{r}}=\frac{f_{r}-f_{0}}{f_{r}}=\alpha \cdot\left(1-e^{-\gamma \cdot \varepsilon_{r 1}^{1+\Delta}}\right)
$$

where $f_{r}$ is the resonant frequency with insertion hole and $f_{0}$ is the resonant frequency in the ideal case, without insertion hole. And the parameters are: 


$$
\begin{array}{ccc}
\alpha=x_{1} \cdot \frac{a^{x_{2}} \cdot b^{x_{3}}}{h^{x_{4}}} \quad \gamma=K_{m} \cdot \frac{a^{3}}{h \cdot b^{2}} \cdot \frac{1}{\alpha} \\
x_{1}=0.1277 \quad x_{2}=1.0464 \quad x_{3}=0.0220 \quad x_{4}=1.0462 \\
\Delta=0.16 \quad K_{m}=0.32
\end{array}
$$

Finally, it is important to note that both equations, Estin and Penaranda-Foix, are also valid when the insertion hole goes through the cavity and it is in the top and in the bottom. In this case the relative error must be doubled. So it is enough to multiply by two equation (74).

\subsection{Measurements}

To probe the validity of the previous expressions, as well as the Generalised Circuital Analysis described along this chapter, some measurements were carried out. These measurements implied two diferent cavities with an insertion hole in the top and in the bottom.

Cavity number 1 has a radius $b=60 \mathrm{~mm}$ and a height $h=20 \mathrm{~mm}$. The insertion hole radius is $a=6.35 \mathrm{~mm}$, and long enough to consider that is under cut-off. Cavity number 2 is exactly the same than number 1 but the external radius is $b=20 \mathrm{~mm}$.

Cavity number 1 is shown in figure $11 a$, where it is open. In figure $11 b$ is shown the same cavity but closed and some samples used to be measured.

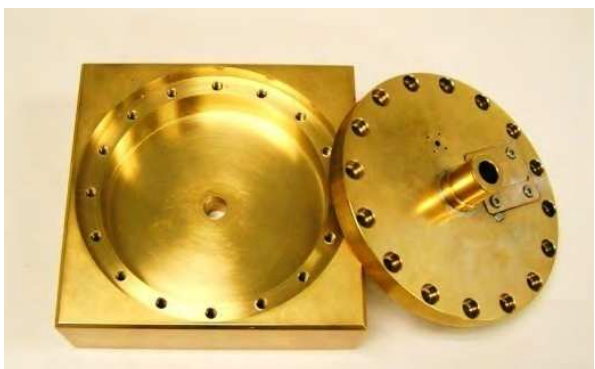

Fig. 11a. Cavity N. 1 open

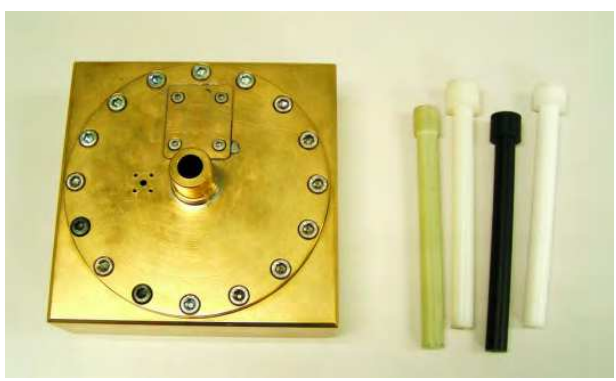

Fig. 11b. Cavity N. 1 closed and samples

The expected resonant frequency deviations is this cavities was simulated and shown in figure 12a. Cavity number 1 has a acpect ratio $a / b=0.1058$ and cavity number 2 aspect ratio is $a / b=0.3175$. Two more aspect ratios have been simulated: $a / b=0.5(b=12.7 \mathrm{~mm})$, that is even smaller than Cavity number 2 , and $a / b=0.04(b=158.75 \mathrm{~mm})$, larger than Cavity number 1 .

Figure 12a shows the predicted frequency deviation by Estin and that predicted by (74), compared with the exact one calculated by circuital analysis following the procedure described along the chapter. And figure $12 \mathrm{~b}$ shows the actual resonant frequency with are without insertion hole.

Table 1 shows the measurements performed with Cavity number $1\left(f_{u}\right.$ is the measured resonant frequency in $\mathrm{GHz}$ and $Q_{u}$ is the measured quality factor) and the calculated permittivities depending the case (note that in all the cases the quality factor used $Q_{d}$ has been calculated with the expression $1 / Q_{d}=1 / Q_{\text {meas }}-1 / Q_{\text {empty }}$ to avoid the wall losses 
effect, in such a way that $Q_{\text {meas }}$ is the mesured quality factor with material and $Q_{\text {empty }}$ is the mesured quality factor without material) for materials M1 (Nylon 66 FG) and M2 (ACETAL): CASE 1: This is the permittivity calculated using the circuital and multimode method, considering the up and down insertion holes. This is going to be considered as the exact and reference case.

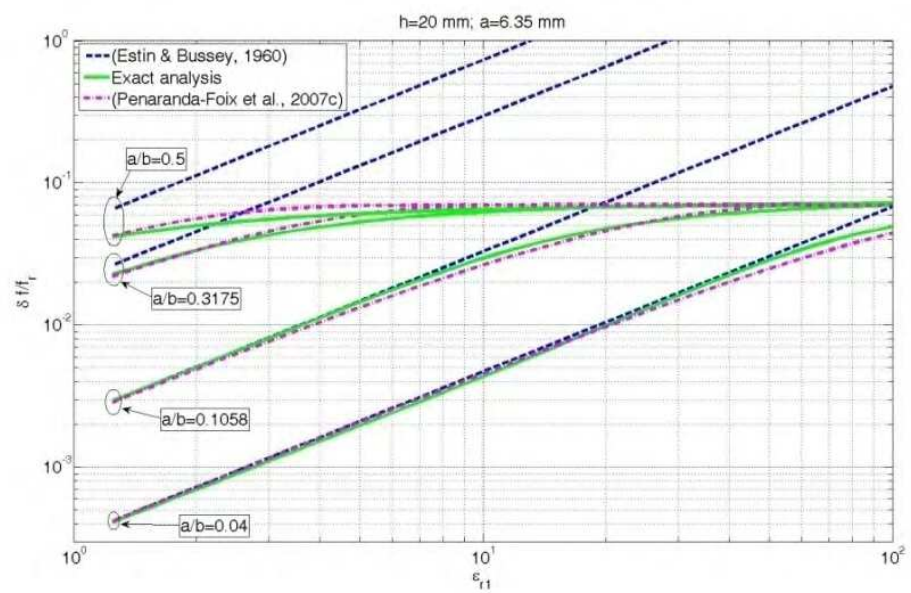

Fig. 12a. Frequency deviation for different permittivities and different aspect ratios

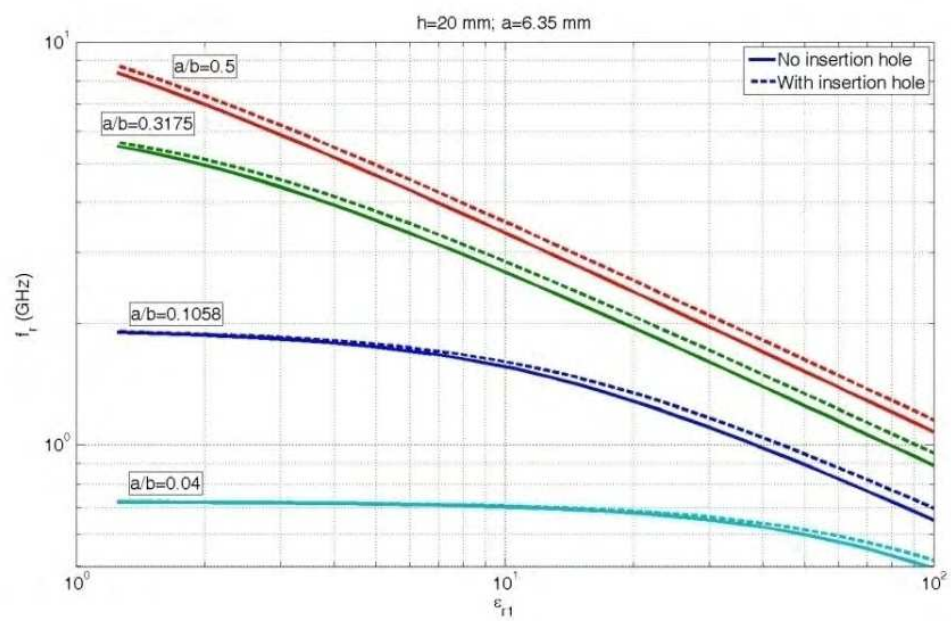

Fig. 12b. Frequency deviation for different permittivities and different aspect ratios.

CASE 2: This is the permittivity computed neglecting the effect of the insertion hole. That is using equation (73) directly.

CASE 3: This is the computed permittivity using the correction proposed by Estin (Estin \& Bussey, 1960).

CASE 4: This is the permittivity using the correction proposed in (74). 
In all the cases the imaginary part of the permittivity has been multiplied by $10^{2}$ and the corrections to the resonant frequency (equations from Estin and (73)) have been applied to the complex resonant frequency, and not only to the resonant frequency- The complex resonant frequency is defined as $\Omega_{r}=f_{r} \cdot(1+j /(2 \cdot Q))$. In Harrington (Harrington, 1961), the reader can find the origin of this concept for resonant problems.

\begin{tabular}{|c|c|c|c|c|c|c|c|c|c|c|}
\hline & & & \multicolumn{2}{|c|}{ CASE 1 } & \multicolumn{2}{c|}{ CASE 2 } & \multicolumn{2}{c|}{ CASE 3 } & \multicolumn{2}{c|}{ CASE 4 } \\
\hline & $\mathrm{f}_{\mathrm{u}}$ & $\mathrm{Q}_{\mathrm{u}}$ & $\varepsilon$ & $\varepsilon^{\prime}$ & $\varepsilon^{\prime}$ & $\varepsilon^{\prime}$ & $\varepsilon$ & $\varepsilon^{\prime}$ & $\varepsilon^{\prime}$ & $\varepsilon{ }^{\prime}$ \\
\hline Air & 1.9157 & 4496 & $60.0274^{4}$ & ------- & ------- & ------- & ------- & ------- & ------- & ------- \\
\hline M1 & 1.8517 & 564.8 & 2.8457 & 4.0922 & 2.5237 & 3.5545 & 2.8291 & 4.0184 & 2.8155 & 3.9760 \\
\hline M2 & 1.8471 & 198.8 & 2.9778 & 12.6630 & 2.6385 & 10.996 & 2.9588 & 12.4362 & 2.9438 & 12.2980 \\
\hline
\end{tabular}

Table 1. Measurements with cavity 1.

It is important to note that the error in CASE 2 in Cavity number 2 is about $12 \%$ respect to the exact, because the insertion hole correction has not been applied. Once the correction is used, even in CASE 3 or CASE 4, errors are reduced to less than $2 \%$.

Table 2 shows exactly the same than before, but measured in a smaller cavity: Cavity number 2.

\begin{tabular}{|c|c|c|c|c|c|c|c|c|c|c|}
\hline & & & \multicolumn{2}{|c|}{ CASE 1 } & \multicolumn{2}{c|}{ CASE 2 } & \multicolumn{2}{c|}{ CASE 3 } & \multicolumn{2}{c|}{ CASE 4 } \\
\hline & $\mathrm{f}_{\mathrm{u}}$ & $\mathrm{Q}_{\mathrm{u}}$ & $\varepsilon^{\prime}$ & $\varepsilon^{\prime}$ & $\varepsilon^{\prime}$ & $\varepsilon^{\prime \prime}$ & $\varepsilon^{\prime}$ & $\varepsilon^{\prime \prime}$ & $\varepsilon$ & $\varepsilon{ }^{\prime}$ \\
\hline Air & 5.8350 & 617.7 & $20.0119^{5}$ & ------- & ------- & ------- & ------- & ------ & ------- & ------- \\
\hline M1 & 4.6045 & 106.5 & 2.8779 & 3.6882 & 2.5410 & 3.1167 & 3.0760 & 4.3551 & 2.8903 & 3.7284 \\
\hline M2 & 4.5979 & 49.00 & 2.8903 & 8.8461 & 2.552 & 7.6725 & 3.0906 & 10.5658 & 2.9032 & 9.0356 \\
\hline
\end{tabular}

Table 2. Measurements with cavity 2.

In this case, and due to a highest aspect ratio in Cavity number 2, the Estin formulae fails. So the error, supposing that CASE 1 is the exact one, errors are about $7 \%$ and $12 \%$ using Estin formulae or no-insertion hole approach. But it reduces again to less than $2 \%$ when using (74).

Finally, it is worth mentioning that all the above results are calculated with an uncertainty of $1.6 \%$ ). This uncertainty has been calculated following the procedure described in (BakerJarvis et al., 1994) or (Bell, 2001). Basically it is based on the calculation of the derivative of the permittivity depending on the variable that affects the accuracy:

$$
\partial \varepsilon_{r 1}=\sqrt{\sum_{i}\left(\frac{\partial \varepsilon_{r 1}}{\partial x_{i}} \cdot \Delta x_{i}\right)^{2}}
$$

where $x_{i}$ are the different dimensions that are involved in the accuracy of the measurement. And to compute the derivatives $\partial \varepsilon_{r 1} / \partial x_{i}$ the three terms Lagrange polynomial interpolation formula was used:

4 This value is not the air permittivity but the real cavity radius calculated from the air resonant frequency.

${ }^{5}$ Once again, this value is not the air permittivity but the real cavity radius calculated from the air resonant frequency. 


$$
f(x)=\sum_{i=0}^{N} a_{i} \cdot h_{i}(x), \quad h_{i}(x)=\prod_{\substack{j=1 \\ j \neq i}}^{N} \frac{x-x_{j}}{x_{i}-x_{j}}
$$

Then three different permittivities are calculated for each source of error in order to compute the interpolation and then the derivative in (76) to calculate the final uncertainty.

\section{Conclusion}

Conclusions are, firstly, that an introduction and a revision of the Generalized Circuital Analysis to solve in a simplest way large electromagnetic problems have been presented.

This method has been successfully applied to a Cylindrical Cavity with insertion hole, creating four different small networks and solving each one to get the Generalized Admittance Matrix (GAM), and joining all together forcing the resonant condition to obtain the complex resonant frequency.

The origin of the problem comes from the deviation of the resonant frequency observed when the insertion hole exists. This deviation can be important if neglected, so a monomode approximation formula has been obtained as well as an optimized equation to estimate the relative error from the measured and actual resonant frequency with and without insertion hole.

The proposed expression improves the only one existing since 1960 by Estin.

\section{References}

Alessandri, Ferdinando; Mongiardo Mauro \& Sorrentino, Roberto (1994). , Rigorous Mode Matching Analysisof Mitered E-Plane Bends in Rectangular Waveguide, IEEE Microwave and Guided Wave Letters, N ${ }^{\circ}$ 12, Vol. 4, pp. 408-410, ISSN 1531-1309

Baker-Jarvis, James; Janezic, Michael D.; Domich, Paul D. \& Geyer, Richard G. (1994). Analysis of an Open-Ended Coaxial Probe with Lift-Off for Nondestructive Testing, IEEE Trans. On Instrumentation and Measurement, vol. 43 No 5, Oct., pp. 711-718, ISSN 0018-9456

Balanis, Constantine A., (1989). Advanced Engineering Electromagnetics, John Wiley and Sons, ISBN 978-0471503163

Bell, Stephanie (2001). Beginner's Guide to Uncertainty of Measurement (Issue 2), National Physical Laboratory (NPL), ISSN 1368-6550. August 1999, Issue 2 with amendments March 2001

Chen, L. F.; Ong, C. K.; Neo, C. P.; Varadan, V.V. \& Varadan V.K., (2004). Microwave Electronics, John Wiley and Sons, ISBN 0-470-84492-2

Collin, Robert E. (1991), Field Theory of Guided Waves, ISBN 978-0879422370, IEEE Press

Collin, Robert E. (1966), Foundations for Microwave Engineering, ISBN 978-0780360310, McGrawHill

Estin, A. J. \& Bussey, H. E. (1960). Errors in Dielectric Measurements Due to a Sample Insertion Hole in a Cavity, IEEE Trans. on MTT, Vol. 8, No. 11, Nov., pp. 650-653, ISSN 0018-9480

Harrington, R. F. (1961). Time-Harmonic Electromagnetic Fields, ISBN 047120806X, New York

Harrington, Roger F. (1967). Matrix Methods for Field Problems, Proceedings of the IEEE, N. 2, Vol. 55, pp. 136-149, ISSN 0018-9219

Harrington, Roger F. (1993). Field Computation by Moment Methods, Wiley-IEEE Press, ISBN 978-07803-1014-8 
Gentili, G.G. (1991). Properties of TE-TM mode-matching techniques, IEEE Trans. on M.T.T., vol. 39, no. 9, Sept., pp. 1669-1673, ISSN 0018-9480

Gentili, G. G. \& Melloni, A. (1996). Generalized Admittance Matrix Analysis of Cylindrical Cavities Coupledby Rectangular Apertures, Journal of Electromagnetic Waves and Applications, № 1,Vol. 10, pp. 129-145, ISSN 0920-5071

Gimeno, Benito \& Guglielmi Marco (1997). Multimode Equivalent Network Representation for Junctions between Coaxial and Circular Waveguides, Int. Journal Microwave MillimeterWave CAE,pp. 180-194, ISSN 1050-1827

Metaxas A.C. \& Meredith R.J. (1988). Industrial Microwave Heating, The Institution of Engineering and Technology, ISBN: 978-0906048894

Penaranda-Foix, Felipe L. (2001). Aplicación de la Teoría de Análisis Circuital Generalizado a la Resolución de Problemas de Difracción Electromagnética, PhD in Universidad Politécnica de Valencia -Valencia (Spain).

Penaranda-Foix, Felipe L. \& Ferrando-Bataller, Miguel (2003). Scattering of Inhomogeneous Cylinders by Circuital Analysis, Microwave and Optical Technology Letters, Vol 39, N. 2, Oct., pp. 155-159, ISSN 0895-2477

Penaranda-Foix, Felipe L.; Catala-Civera, José M.; Canos-Marin, Antoni J. \& Plaza-Gonzalez, Pedro J. (2007a). Solving Cylindrically-Shaped Waveguides Partially-Filled with Isotropic Materials by Modal Techniques. Proceedings of 11th. AMPERE 2007, Vol. 1, pp. 67-70, ISBN 978-973-759-333-7, Oradea (Romania), 3-6 September

Penaranda-Foix, Felipe L.; Catala-Civera, Jose M.; Canos-Marin, Antoni J. \& Garcia-Banos, Beatriz (2007b). Frequency Deviation Due to a Sample Insertion Hole in a Cylindrical Cavity by Circuital Analysis. Proceedings of the IMS2007, Vol. 1 pp. 1683-1686, ISBN 14244-0688-9/07, Honolulu (USA)

Penaranda-Foix, Felipe L.; Catala-Civera, Jose M.; Canos-Marin, Antoni J. \& Garcia-Banos, Beatriz (2007c). Practical expression for frequency deviation due to insertion hole in cylindrical cavity, Electronics Letters, Vol 43, N. 24, 22nd Nov., pp. 1338-1340, ISSN 00135194

Penaranda-Foix, Felipe L.; Catala-Civera, Jose M.; Canos-Marin, Antoni J. \& Garcia-Banos, Beatriz (2009). Circuital analysis of a coaxial re-entrant cavity for performing dielectric measurement. Proceedings of the IMS2009, Vol. pp. 1309-1312, ISBN 978-1-4244-2804-5, Boston (USA)

Pozar, David M. (1990), Microwave Engineering, ISBN 978-0471448785, Addison-Wesley Publishing Company, Inc.

Ramo, Simon; Whinnery, John R. \& Duzer, Theodore van (1994). Fields and waves in communication electronics, John Wiley \& Sons, Inc., ISBN 0471585513

Rebollar, Jesus M.; Esteban, J. \& Page, J. E. (1994). Fullwave Analysis of Three and Four-Port Rectangular Waveguide Junctions, IEEE Trans. on M.T.T., N ${ }^{\circ}$ 2, Vol. 42, pp. 256-263, ISSN 0018-9480

Valero-Nogueira, Alejandro (1997). Resolución de Problemas Electromagnéticos Complejos Mediante Análisis Circuital Generalizado, PhD in Universidad Politécnica de Valencia-Valencia (Spain).

Wexler, A. (1967). Solution of waveguide discontinuities by modal analysis, IEEE Trans. MTT, Vol. 15, N. 9, pp.508-517, ISSN 0018-9480 


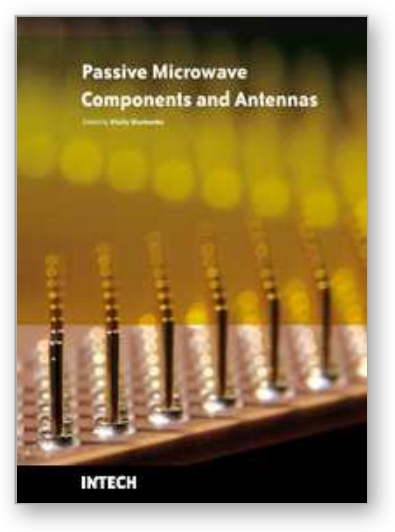

\author{
Passive Microwave Components and Antennas \\ Edited by Vitaliy Zhurbenko
}

ISBN 978-953-307-083-4

Hard cover, 556 pages

Publisher InTech

Published online 01, April, 2010

Published in print edition April, 2010

Modelling and computations in electromagnetics is a quite fast-growing research area. The recent interest in this field is caused by the increased demand for designing complex microwave components, modeling electromagnetic materials, and rapid increase in computational power for calculation of complex electromagnetic problems. The first part of this book is devoted to the advances in the analysis techniques such as method of moments, finite-difference time- domain method, boundary perturbation theory, Fourier analysis, mode-matching method, and analysis based on circuit theory. These techniques are considered with regard to several challenging technological applications such as those related to electrically large devices, scattering in layered structures, photonic crystals, and artificial materials. The second part of the book deals with waveguides, transmission lines and transitions. This includes microstrip lines (MSL), slot waveguides, substrate integrated waveguides (SIW), vertical transmission lines in multilayer media as well as MSL to SIW and MSL to slot line transitions.

\title{
How to reference
}

In order to correctly reference this scholarly work, feel free to copy and paste the following:

Felipe L. Penaranda-Foix and Jose M. Catala-Civera (2010). Circuital Analysis of Cylindrical Structures Applied to the Electromagnetic Resolution of Resonant Cavities, Passive Microwave Components and Antennas, Vitaliy Zhurbenko (Ed.), ISBN: 978-953-307-083-4, InTech, Available from:

http://www.intechopen.com/books/passive-microwave-components-and-antennas/circuital-analysis-ofcylindrical-structures-applied-to-the-electromagnetic-resolution-of-resonant-c

\section{INTECH}

open science | open minds

\section{InTech Europe}

University Campus STeP Ri

Slavka Krautzeka 83/A

51000 Rijeka, Croatia

Phone: +385 (51) 770447

Fax: +385 (51) 686166

www.intechopen.com

\section{InTech China}

Unit 405, Office Block, Hotel Equatorial Shanghai

No.65, Yan An Road (West), Shanghai, 200040, China 中国上海市延安西路65号上海国际贵都大饭店办公楼 405 单元

Phone: $+86-21-62489820$

Fax: +86-21-62489821 
(C) 2010 The Author(s). Licensee IntechOpen. This chapter is distributed under the terms of the Creative Commons Attribution-NonCommercialShareAlike-3.0 License, which permits use, distribution and reproduction for non-commercial purposes, provided the original is properly cited and derivative works building on this content are distributed under the same license. 